\title{
Hybrid Tabu Algorithm for the Synthesis and Fabrication of Fiber Bragg Gratings
}

\author{
Nam Quoc Ngo ${ }^{1}$ and Ruitao Zheng ${ }^{2}$ \\ ${ }^{1}$ Nanyang Technological University \\ ${ }^{2}$ Avago Technologies Manufacturing (Singapore) Pte. Ltd. \\ Singapore
}

\section{Introduction}

Fiber Bragg gratings (FBGs) are widely employed as optical filters for performing various functions such as add/drop multiplexers, dispersion compensators and multiplexers/demultiplexers for use in optical communication systems and optical sensors because of a number of advantages that include low insertion loss, low polarization sensitivity, all-fiber geometry, compactness, easy fabrication and low cost (Kashyap, 1999; Othonos \& Kalli, 1999). In addition, the technology of ultraviolet (UV) photoinduced FBGs is quite mature to allow the fabrication of a wide variety of FBGs with complex characteristics. To meet the increasing demand for large capacity of the next generation of optical communication systems (i.e., wavelength division multiplexing (WDM) networks) and optical sensors, there is an important need for a powerful design tool that can be used for the synthesis or design of FBGs from the specified frequency responses that can be practically realized. This synthesis or inverse problem of determining a FBG structure from a given frequency response (i.e., magnitude and phase responses) is common in many application areas. The design tool must be efficient and reliable to enable the synthesis of FBG-based filters with prescribed frequency responses, depending on the application requirements. That is, the design tool must be able to determine the index modulation profile and hence the structure of an FBG from a given frequency response. In addition, the design tool must be powerful enough for use in the diagnosis or characterization during and after fabrication of an FBG. Although several synthesis methods such as those based on the layer-peeling algorithm have been proposed for the synthesis of FBGs from the specified frequency responses, the index modulation profiles of the synthesized FBGs are not optimized and are often complex, making practical realization difficult (Feced et al., 1999; Poladian, 2000; Skaar et al., 2001; Rosenthal \& Horowitz, 2003). To overcome this problem, optimization methods have been demonstrated as an attractive approach because it allows weighting mechanisms to be incorporated into the desired frequency response of an FBGbased filter to be synthesized, resulting in an optimum and practically realizable index modulation profile of the FBG structure. Furthermore, the optimization algorithms also allow additional constraints to be included in the weighting mechanisms of the specified frequency response to suit certain condition(s) or constraint(s) of a particular fabrication system. In optimization, the FBG synthesis problems are formulated as nonlinear objective

Source: Local Search Techniques: Focus on Tabu Search, Book edited by: Wassim Jaziri, ISBN 978-3-902613-34-9, pp. 278, October 2008, I-Tech, Vienna, Austria 
functions and the optimized solution of the FBG design is obtained by finding the global optimum of the objective function. Although several global optimization algorithms such as the global genetic algorithm (GA) (Skaar \& Risvik, 1998; Gill et al., 2004; Cheng \& Lo, 2004), the global simulated annealing (SA) (Dong, Azana \& Kirk, 2003) and the local optimization method such as the Levenberg-Marquardt algorithm (Plougmann \& Kristensen, 2004) have been applied to solving the FBG synthesis problems, the obtained solutions are, in general, not optimum, making practical implementation difficult. This is because, in general, it is important to employ a global optimization algorithm to solve an FBG synthesis problem to ensure that a global optimum can be obtained; however, convergence of the global optimization method is normally not as good as that of a local optimization algorithm. Unfortunately local optimization methods also cannot easily solve the FBG synthesis problems due to the multimodal and ill-conditioned character of the nonlinear objective functions. To improve the convergence of the global optimization algorithm, a hybrid algorithm combining a global optimization algorithm and a local optimization algorithm has been shown to be a better approach for the synthesis and fabrication of FBG-based bandpass filters (Zheng et al., 2004; Ngo et al., 2007). This is the motivation of this chapter which describes the use of a hybrid Tabu algorithm for the synthesis and fabrication of FBGbased bandpass filters and linear phase filters from the given frequency responses. (Ngo et al., 2004; Zheng et al., 2005) presented the first reports of employing the standard and improved Tabu search algorithms (Glover \& Laguna, 1998; Chelouah \& Siarry, 2000) for the synthesis of FBG-based bandpass filters and linear phase filters. The hybrid Tabu algorithm is a two-tier search that employs a global optimization algorithm (i.e., a staged continuous Tabu search (SCTS) algorithm (Zheng et al., 2005) which performs better than the standard Tabu search (Ngo et al., 2004)) and a local optimization algorithm (i.e., the Quasi-Newton method (Shanno, 1970) which has high efficiency in solving multimodal nonlinear optimization problems). First, the global SCTS algorithm, in which a dynamic mechanism for weighting of different requirements of the magnitude and phase responses is employed to enhance the optimization efficiency, is used to find a "promising" FBG structure that has a frequency response as close as possible to the target one. The local Quasi-Newton algorithm is then applied to further optimize this "promising" FBG structure obtained from the global SCTS algorithm to obtain the final optimum solution. To demonstrate the effectiveness of the hybrid Tabu method which has high convergence rate and high reliability, the synthesis and fabrication of several FBG-based bandpass filters and linear phase filters for application in optical communications are presented in this chapter. It is worth mentioning that other stochastic search algorithms of various types have also been applied to the design of FBGs: the Nelder-Mead Simplex hill climbing algorithm (Caucheteur et al., 2004) and particle swarm optimization (Baskar et al., 2005a), evolutionary strategies such as the covariance matrix adapted evolution (Baskar et al., 2005b; Baskar et al., 2006), and a multi-objective evolutionary algorithm (Manos \& Poladian, 2005).

\section{Transfer matrix method for solving non-uniform Bragg gratings}

\subsection{Theory of fiber Bragg grating}

There are two main types of fiber Bragg gratings (FBGs), namely, uniform FBG (which has equal grating periods) and non-uniform FBG (which has unequal grating periods). Singlemoded FBGs are considered here because they are commonly used in many areas of optics and photonics. For ease of discussion, the uniform FBG is considered in this section. An FBG 
is essentially a filter written into the core of a segment of optical fiber via the interference of two ultraviolet (UV) beams from a UV laser (see Fig. 1). The interference pattern forms a periodic refractive index change (or index perturbation) along the longitudinal direction of the fiber. Due to the index change, the FBG acts as a series of reflectors, reflecting back a small amount of the input light with wavelength components that are close to the Bragg wavelength.

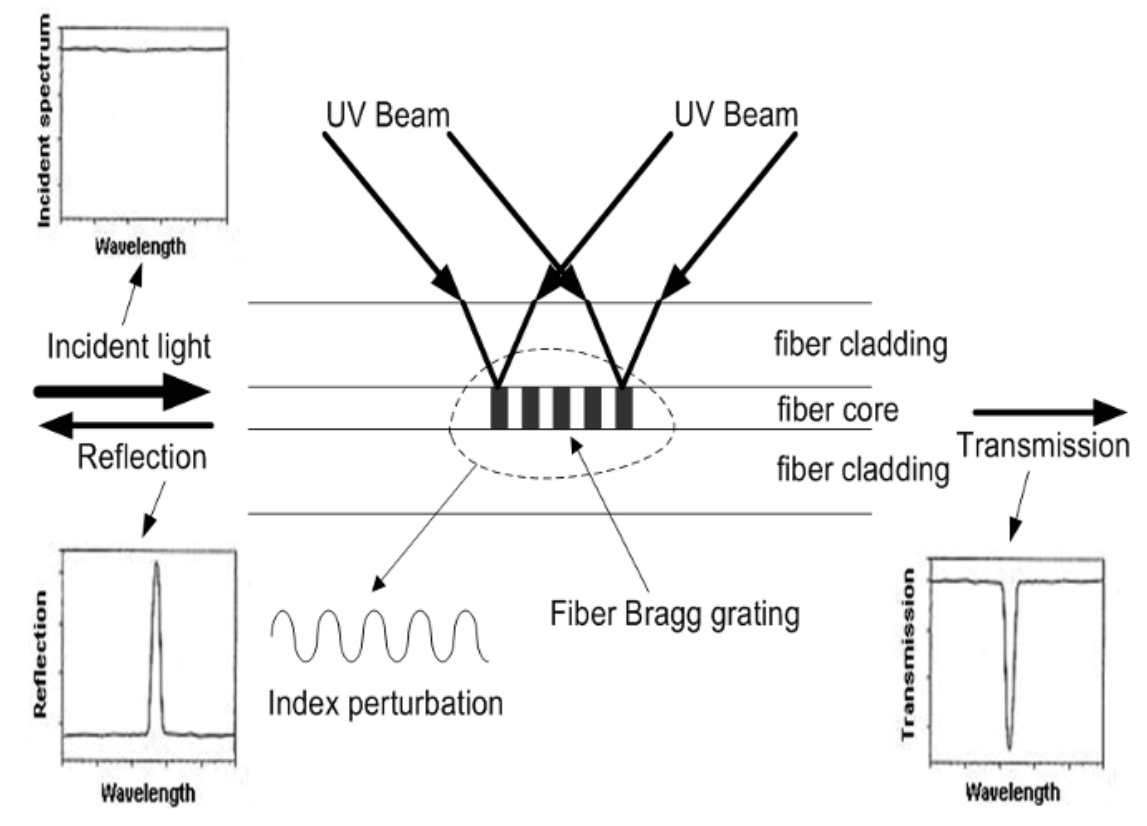

Fig. 1. Schematic diagram of a typical interferometric system used for the fabrication of fiber Bragg gratings (FBGs). The FBG shown here is of a uniform type with equal grating periods.

The Bragg wavelength, $\lambda_{B}(z)$, of a uniform FBG is the wavelength that fulfills the Bragg condition:

$$
\lambda_{B}(z)=2 \bar{n}_{\mathrm{eff}}(z) \Lambda(z)
$$

where $z$ is the longitudinal coordinate along the length of the grating and $\Lambda(z)$ is the perturbation period or grating period (see Fig. 2). The average effective refractive index on the grating is defined as (see Fig. 2)

$$
\bar{n}_{\mathrm{eff}}(z)=n_{0}+\Delta n_{\mathrm{dc}}(z)
$$

where $n_{0}$ is the effective index without UV exposure and $\Delta n_{\mathrm{dc}}(z)$ is the " $\mathrm{dc}$ " (or average) index change spatially averaged over the grating. Fig. 2 shows the quasi-sinusoidal profile of the effective index, $n_{\text {eff }}(z)$, which is described by 


$$
n_{\text {eff }}(z)=\bar{n}_{\mathrm{eff}}(z)+\Delta n_{\mathrm{ac}}(z) \cdot f(z) \cdot \cos \left[\frac{2 \pi z}{\Lambda(z)}+\phi(z)\right]
$$

where $\Delta n_{\mathrm{ac}}(z)$ is the "ac" index change (i.e., index modulation), $f(z)$ is the normalized apodization function, and $\phi(z)$ is the chirp profile of the grating. Putting Eq. (2) into Eq. (3), the index perturbation, $\delta n_{\mathrm{eff}}(z)$, is given by

$$
\delta n_{\mathrm{eff}}(z)=n_{\mathrm{eff}}(z)-n_{0}=\Delta n_{\mathrm{dc}}(z)+\Delta n_{\mathrm{ac}}(z) \cdot f(z) \cdot \cos \left[\frac{2 \pi z}{\Lambda(z)}+\phi(z)\right]
$$

Thus Eq. (4) shows that the optical properties of an FBG are essentially determined by the variation of the index perturbation along the grating length.

Optical waveguide

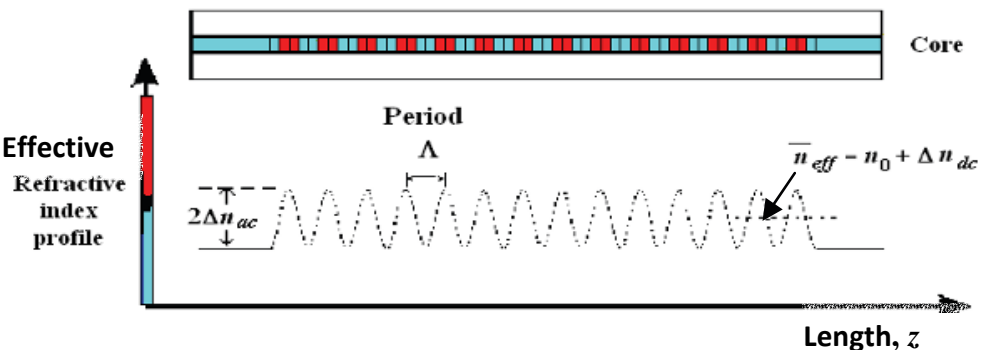

Fig. 2. Schematic showing an example of an FBG and its refractive index profile. The FBG shown here is of a uniform type with equal grating periods.

Figure 3 shows the index perturbation profiles of a uniform FBG, an apodized FBG with variable-dc index change, and an apodized FBG with zero-dc index change, which are considered in this work. Other types of index perturbation profiles such as chirped FBG, phase-shifted FBG and super-structured FBG can also be employed, depending on the desired filter responses (Erdogan, 1997).

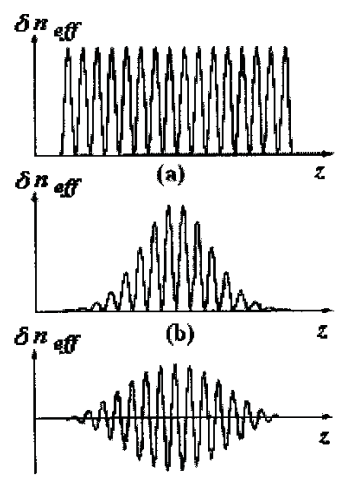

(c)

Fig. 3. Index modulation profiles of the types of FBGs considered here. (a) A uniform FBG with a constant dc index change. (b) An apodized FBG with variable-dc index change. (c) An apodized FBG with zero-dc index change. 
The length of the FBG typically ranges from several millimeters to tens of centimeters, depending on the desired filter responses. Because there are tens of thousands of these perturbation periods of index changes or reflectors in a row, an FBG-based filter generally has a near-squared reflective magnitude response. In the reflection mode, the reflective magnitude response has a very narrow bandpass response (with a typical 3-dB bandwidth of $0.2 \mathrm{~nm}$ or $25 \mathrm{GHz}$ in the $1550 \mathrm{~nm}$ wavelength window) at the Bragg wavelength; whereas in the transmission mode, the transmissive magnitude response has a very narrow notch response at the Bragg wavelength (see Fig. 1). The phase-mask technique is one of the most effective methods used in the fabrication of FBGs because it employs a simple diffractive optical element (or a phase mask) to spatially modulate the UV beam (Kashyap, 1999; Othonos \& Kalli, 1999). Compared with two other main types of optical filters, namely, thin film filters and arrayed waveguide gratings, FBG-based filters have many unique advantages such as low loss, low polarization sensitivity, simple all-fiber geometry, easy fabrication, and low cost.

\subsection{Transfer matrix method for solving non-uniform fiber Bragg gratings}

The coupled-mode theory has been widely used for the analysis of FBGs because it allows one to determine the effect of the grating structure on the frequency response (i.e., magnitude and phase responses) (Erdogan, 1997; Hill \& Meltz, 1997; Kashyap, 1999; Othonos \& Kalli, 1999). A uniform FBG is the simplest type of FBG to design and fabricate because it simply has equal grating periods and a constant modulation depth of the refractive index (see Fig. 1, Fig. 2, Fig. 3(a)). The uniform FBG can be easily designed because an analytical solution to the coupled-mode equation can be easily obtained. However, due to the finite length of the uniform FBG, the roll offs on the two edges of the reflective magnitude response are not sharp enough due to the presence of sidelobes on both sides of the bandpass response. This drawback has limited the application of uniform FBGs. This limitation can be overcome by using a non-uniform FBG which has a more squared reflective magnitude response with much smaller amplitudes of the sidelobes. However, analytical solutions to the coupled-mode equations describing the non-uniform FBGs cannot be easily obtained. The transfer matrix method (TMM) has been widely employed for solving the non-uniform FBGs due to its high computational efficiency and high reliability (Erdogan, 1997; Kashyap, 1999). The TMM method allows the magnitude and phase responses of a non-uniform FBG to be easily obtained with reasonably high accuracy. In TMM, a non-uniform FBG can be modeled using two methods, depending on the requirements of the magnitude and phase responses: (1) A non-uniform FBG model using the cascade of serially-connected uniform sub-gratings (this model is sufficient for obtaining the magnitude response when the phase response is not important); and (2) A non-uniform FBG model using the cascade of serially-connected apodized sub-gratings (this model must be used when both the magnitude and phase responses are important). These two methods are described below.

\subsubsection{A non-uniform FBG model using the cascade of uniform sub-gratings}

When only the magnitude response of a filter (whose phase response is not important) is required to be designed, the TMM method can be used to model a non-uniform FBG as the cascade of serially-connected uniform sub-gratings. The non-uniform FBG can be divided into a number of serially-connected $N$ uniform sub-gratings or sections (as shown in Fig. 4). 
Each uniform sub-grating section can be described by an analytic transfer matrix. The transfer matrix for the entire non-uniform FBG structure can be obtained by simply multiplying the individual transfer matrices of the uniform sub-gratings.

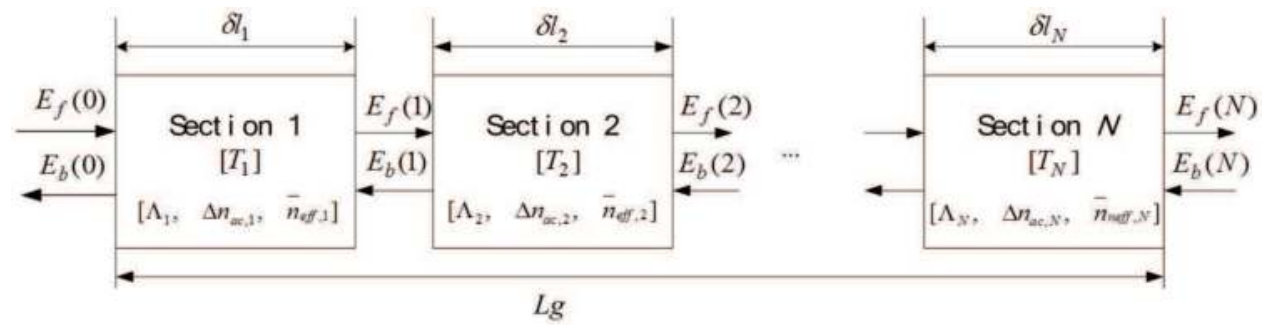

Fig. 4. Schematic diagram of a non-uniform FBG model based on the cascade of seriallyconnected uniform sub-gratings.

Each uniform sub-grating section is described by an index perturbation defined in Eq. (4). Note that Eq. (4) is a general equation describing the index perturbation of all kinds of FBGs. To apply Eq. (4) to each uniform sub-grating section, the parameters in this equation are redefined as follows. A uniform sub-grating section requires $f(z)=1$ and $\phi(z)=0$ in Eq. (4). Putting these conditions into Eq. (4) gives a simpler index perturbation, $\delta n_{\text {eff }}(z)$, for each uniform sub-grating which is given by

$$
\delta n_{\mathrm{eff}}(z)=\Delta n_{\mathrm{dc}}(z)+\Delta n_{\mathrm{ac}}(z) \cdot \cos (2 \pi z / \Lambda(z))
$$

Equation (5) is schematically shown in Fig. 3(a). In Fig. $4, E_{\mathrm{f}}(j ; \lambda)$ and $E_{\mathrm{b}}(j ; \lambda)$ are the complex electric fields of the forward and backward propagation waves, respectively, describing the $j^{\text {th }}$ section. Also, $\delta l_{j}, \Lambda_{j}, \Delta n_{\mathrm{ac}, j}$ and $\bar{n}_{\mathrm{eff}, j}$ (and hence $\Delta n_{\mathrm{dc}, j}$ according to Eq. (2)), which are the parameters to be optimized in this particular model, are the length, period, 'ac' index change (i.e., index modulation) and average effective index of the $j^{\text {th }}$ section, respectively. In this model, the parameters that are not optimized are $\Lambda_{j}$ and $\Delta n_{\mathrm{dc}, j}(z)=\Delta n_{\mathrm{dc}, j}$ (and hence $\bar{n}_{\mathrm{eff}, j}$ according to Eq. (2)), which are the period and the "dc" index change, respectively, and they are fixed or constant values. $L_{\mathrm{g}}$ is the total length of the non-uniform FBG. The designer has the choice of choosing which of these four (4) variables (i.e., $\delta l_{j}, \Lambda_{j}, \Delta n_{\mathrm{ac}, j}$ and $\bar{n}_{\mathrm{eff}, j}$ ) are to be optimized, depending on the fabrication condition(s) or constraint(s) of a particular fabrication system. The complex electric fields at the input ports $\left(E_{\mathrm{f}}(0 ; \lambda), E_{\mathrm{b}}(0 ; \lambda)\right)$ and output ports $\left(E_{\mathrm{f}}(N ; \lambda), E_{\mathrm{b}}(N ; \lambda)\right)$ of the nonuniform FBG are described by

$$
\left[\begin{array}{c}
E_{\mathrm{f}}(0 ; \lambda) \\
E_{\mathrm{b}}(0 ; \lambda)
\end{array}\right]=T \cdot\left[\begin{array}{c}
E_{\mathrm{f}}(N ; \lambda) \\
E_{\mathrm{b}}(N ; \lambda)
\end{array}\right] ; \quad T=\prod_{j=1}^{N} T_{j}
$$

where $T_{j}=\left[\begin{array}{ll}T_{11} & T_{12} \\ T_{21} & T_{22}\end{array}\right]$ is the $2 \times 2$ transfer matrix of the $j^{\text {th }}$ section. The elements of the transfer matrix are defined as 


$$
\begin{gathered}
T_{11}=\cosh \left(S_{j} \delta l_{j}\right)-\frac{i \hat{\sigma}_{j}}{S_{j}} \sinh \left(S_{j} \delta l_{j}\right), \quad T_{22}=\cosh \left(S_{j} \delta l_{j}\right)+\frac{i \hat{\sigma}_{j}}{S_{j}} \sinh \left(S_{j} \delta l_{j}\right) \\
T_{12}=-\frac{i \kappa_{j}}{S_{j}} \sinh \left(S_{j} \delta l_{j}\right), \quad T_{21}=\frac{i \kappa_{j}}{S_{j}} \sinh \left(S_{j} \delta l_{j}\right)
\end{gathered}
$$

where $i=\sqrt{-1}$. The 'ac' coupling coefficient, $\kappa_{j}$, is defined as

$$
\kappa_{j}=i \frac{\pi}{\lambda} \Delta n_{\mathrm{ac}, j}
$$

where $\lambda$ is the optical wavelength. The ' $\mathrm{dc}^{\prime}$ coupling coefficient, $\hat{\sigma}_{j}$, is defined as

$$
\hat{\sigma}_{j}=\delta_{j}+\sigma_{j}
$$

where $\delta_{j}=\beta_{j}-\pi / \Lambda_{j}=2 \pi \bar{n}_{\text {eff, }},\left(1 / \lambda_{j}-1 / \lambda_{\mathrm{B}, j}\right)$ is the detuning parameter at the wavelength $\lambda_{j}, \quad \beta_{j}$ is the propagation constant, $\lambda_{\mathrm{B}, j}=2 \bar{n}_{\mathrm{eff}, j} \Lambda_{j}$ is the Bragg wavelength, and $\bar{n}_{\mathrm{eff}, j}=n_{0}+\Delta n_{\mathrm{dc}, j}$ (see Eq. (2)). The coefficient $\sigma_{j}$ is defined as

$$
\sigma_{j}=\frac{2 \pi}{\lambda_{j}} \Delta n_{\mathrm{dc}, j}
$$

where $\Delta n_{\mathrm{dc}, j}$ is given in Eqs. (2) and (4). $S_{j}$ in Eqs. (7) and (8) is defined as

$$
S_{j}^{2}=\kappa_{j}^{2}-\hat{\sigma}_{j}^{2}
$$

Applying the boundary condition $E_{\mathrm{b}}(N ; \lambda)=0$ (i.e., there is no input to the right side of the FBG), the reflection frequency response $\rho(\lambda)$ and the transmission frequency response $t_{x}(\lambda)$ are given by

$$
\begin{array}{r}
\rho(\lambda)=\frac{E_{\mathrm{b}}(0 ; \lambda)}{E_{\mathrm{f}}(0 ; \lambda)} \\
t_{x}(\lambda)=\frac{E_{\mathrm{f}}(N ; \lambda)}{E_{\mathrm{f}}(0 ; \lambda)}
\end{array}
$$

The reflection magnitude response and the transmission magnitude response are simply given by $|\rho(\lambda)|^{2}$ and $\left|t_{x}(\lambda)\right|^{2}$, respectively. The reflection phase response and the transmission phase response are defined as $\arg [\rho(\lambda)]$ and $\arg \left[t_{x}(\lambda)\right]$, respectively, where arg stands for argument. We here consider only the reflection frequency response (i.e., magnitude and phase responses) or Eq. (13) because we are interested in the bandpass 
response of the filter. The transmission magnitude response will give a bandstop or notch response. The delay time, $\tau_{\rho}(\lambda)$, of light reflected off a grating corresponds to the phase change of $\rho(\lambda)$ relative to the optical wavelength $\lambda$, and is given by (Erdogan, 1997)

$$
\tau_{\rho}(\lambda)=-\frac{\lambda^{2}}{2 \pi c} \cdot \frac{d \theta_{\rho}}{d \lambda}
$$

where $\theta_{\rho}=\arg [\rho(\lambda)]$ is the phase response of $\rho(\lambda)$ and $c$ is the speed of light in vacuum. The dispersion of the grating, $d_{\rho}(\lambda)$, is therefore given by

$$
d_{\rho}(\lambda)=\frac{d \tau \rho}{d \lambda}=-\frac{\lambda^{2}}{2 \pi c}\left(\frac{d^{2} \theta_{\rho}}{d \lambda^{2}}+\frac{2}{\lambda} \cdot \frac{d \theta_{\rho}}{d \lambda}\right)
$$

The non-uniform FBG model based on the cascade of serially-connected uniform subgratings described here is useful for use in the design of the magnitude response (but not the phase response) of a bandpass filter. The model will be used for the design and fabrication of bandpass filters (where the phase responses are not of interest) which are described in Section 4.3. When both the magnitude and phase responses of the filter (e.g., a linear phase filter) are required to be designed, the non-uniform FBG model based on the cascade of serially-connected apodized sub-gratings must be used and it is described below.

\subsubsection{A non-uniform FBG model using the cascade of apodized sub-gratings}

When both the magnitude and phase responses of a filter are required to be designed, the TMM method can be used to model a non-uniform FBG as the cascade of serially-connected apodized sub-gratings. The non-uniform FBG can be divided into a number of seriallyconnected $N$ apodized sub-gratings or sections (as shown in Fig. 5). Each apodized sub-grating section can be described by an analytic transfer matrix. The transfer matrix for the entire non-uniform FBG structure can be obtained by simply multiplying the individual transfer matrices of the apodized sub-gratings.

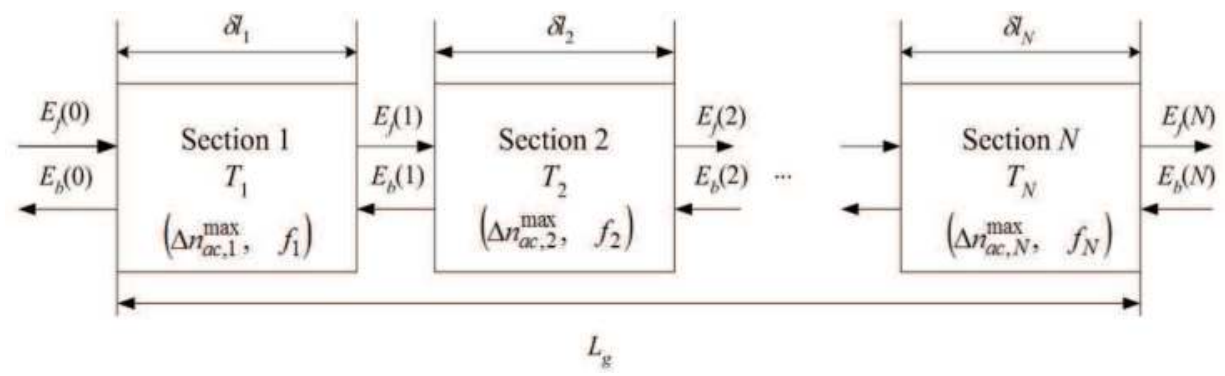

Fig. 5. Schematic diagram of a non-uniform FBG model based on the cascade of seriallyconnected apodized sub-gratings.

Each apodized sub-grating section is described by the index perturbation defined in Eq. (4). Note that Eq. (4) is a general equation describing the index perturbation of all kinds of FBGs. To apply Eq. (4) to each apodized sub-grating section, the parameters in this equation are re- 
defined as follows. An apodized sub-grating section requires $\phi(z)=0$ in Eq. (4). Putting this condition into Eq. (4) gives a simpler index perturbation, $\delta n_{\mathrm{eff}}(z)$, for each apodized subgrating which is given by

$$
\delta n_{\mathrm{eff}}(z)=\Delta n_{\mathrm{dc}}(z)+\Delta n_{\mathrm{ac}}(z) \cdot f(z) \cdot \cos (2 \pi z / \Lambda(z))
$$

Equation (17) is schematically shown in Fig. 3(b). In Fig. 5, $E_{\mathrm{f}}(j ; \lambda)$ and $E_{\mathrm{b}}(j ; \lambda)$ are the complex electric fields of the forward and backward propagation waves, respectively, describing the $j^{\text {th }}$ section. Also, $\delta l_{j}, \Delta n_{\mathrm{ac}, j}$ and $f_{j}$, which are the parameters to be optimized in this particular model, are the length, 'ac' index change (i.e., index modulation) and apodization function of the $j^{\text {th }}$ section, respectively. In this model, the parameters that are not optimized are $\Lambda_{j}$ and $\Delta n_{\mathrm{dc}, j}(z)=\Delta n_{\mathrm{dc}, j}$ (and hence $\bar{n}_{\mathrm{eff}, j}$ according to Eq. (2)), which are the period and the "dc" index change, respectively, and they are fixed or constant values. $L_{\mathrm{g}}$ is the total length of the non-uniform FBG. The designer has the choice of choosing which of these five (5) variables (i.e., $\delta l_{j}, \Delta n_{\mathrm{ac}, j}, f_{j}, \Lambda_{j}$ and $\Delta n_{\mathrm{dc}, j}$ ) are to be optimized, depending on the fabrication condition(s) or constraint(s) of a particular fabrication system. Unlike a uniform FBG (i.e., no apodization) which has sidelobes of about $-10 \mathrm{~dB}$ in the reflective magnitude response, applying apodization to a non-uniform FBG can significantly suppress the sidelobes to $>-40 \mathrm{~dB}$ in the reflective magnitude response. Listed below are several commonly used apodization functions which can be applied to each sub-grating section

$$
\begin{aligned}
& \text { Raised cosine: } f_{j}(z)=\frac{1}{2}\left\{1+\cos \left[\frac{\pi\left(z-\delta l_{j} / 2\right)}{\delta l_{j}}\right]\right\} \\
& \text { Gaussian: } f_{j}(z)=\exp \left[-\frac{(4 \ln 2)\left(z-\delta l_{j} / 2\right)^{2}}{\left(\delta l_{j} / 3\right)^{2}}\right]
\end{aligned}
$$

$$
\text { Sine: } f_{j}(z)=\sin \left(\pi z / \delta l_{j}\right)
$$

$$
\text { Quadratic sine: } f_{j}(z)=\sin ^{2}\left(\pi z / \delta l_{j}\right)
$$

Note that the only difference between a uniform sub-grating (as described in Section 2.2.1) and an apodized sub-grating is that the former has an index perturbation described by Eq. (5) while the latter has an index perturbation described by Eq. (17). In general, the number of apodized sub-gratings is smaller than the number of uniform sub-gratings to achieve a particular desired frequency response. Thus Eqs. (6)-(12) are also applicable to the apodized sub-gratings by putting the right condition defined in Eq. (17) into Eqs. (6)-(12). Putting Eq. (17) into Eqs. (6)-(12), the reflection frequency response, $\rho(\lambda)$, and the transmission frequency response, $t_{x}(\lambda)$, can be computed using Eqs. (13) and (14), respectively. Also, the 
delay time, $\tau_{\rho}(\lambda)$, of light reflected off a grating can be determined using Eq. (15) and the dispersion of the grating, $d_{\rho}(\lambda)$, can be calculated using Eq. (16). This model will be used for the design of FBG-based linear phase filters which is described in Section 4.4.

\section{Staged continuous Tabu search algorithm}

\subsection{Review of Tabu search algorithm}

Tabu search (TS) is an iterative search method originally developed by Glover and Laguna (Glover \& Laguna, 1998) which has been successfully applied to a variety of combinatorial global optimization problems (Ho et al., 2001; Machado et al., 2001; Chelouah \& Siarry, 2000). The TS algorithm has been used for the synthesis of FBG-based linear phase filters using the non-uniform FBG model based on the cascade of serially-connected apodized subgratings (Ngo et al., 2004). A good analogy of how TS works is mountain climbing, where the climber must selectively remember key elements of the path traveled (using adaptive memory) and must be able to strategize choices along the way (using responsive exploration). A rudimentary form of this algorithm can be summarized as follows. It starts from an initial solution $s$ that is randomly selected. From this current solution $s$, a set of neighbors, called $s$, is generated by pre-defining such a set of "moves" or perturbations of the current solution. To avoid the endless reiterative cycle, the neighbors of the current solution, which belong to a subsequently defined "tabu list", are systematically eliminated. The objective function to be minimized is then evaluated for each generated solution s', and the best neighborhood of $s$ becomes the new current solution s' even if it is worse than $s$. The "move" that generates the new current solution will also be stored in the tabu list, which is circular. When the tabu list is full, it is updated by eliminating the previous estimated solution. Then a new "iteration" is performed; the previous procedure is repeated by starting from the new current solution until a pre-defined stopping condition is satisfied. The TS algorithm has a small probability of becoming trapped in a local optimum. Compared to other traditional methods such as the genetic algorithm (GA) and the simulated annealing (SA), the TS algorithm has one unique advantage in that it can also be organized to take advantage of problem-specific information and thus has higher convergence velocity as well as higher level of reliability. TS also includes "candidate list strategies" for generating and sampling neighbors. These candidate list strategies are very important because often only a relatively small subset of neighbors is generated at any given iteration, especially when a large number of neighborhoods is used, as in the case of multi-variable problems whose neighbors are generated in a multi-dimensional space.

\subsection{Review of continuous Tabu search algorithm}

As an enhancement to the TS algorithm described above in terms of higher convergence velocity and higher level of reliability, a continuous Tabu search (CTS) algorithm, which employs a special "candidate list strategy" to generate neighbors, has been proposed for the optimization of nonlinear objective functions (Siarry \& Berthiau, 1997). In this method, the solution space is divided into several regions. Neighbors are generated in these regions and the remainder of the method consists of an elementary form of TS that uses only the simple tabu list construction as described in Section 3.1. A brief review of the CTS algorithm is described here because it will be used for the development of a staged continuous search (SCTS) algorithm in the subsequent section. For the following optimization problem: 


$$
\min _{s \in \Psi^{k}}[\Phi(s)]
$$

where $\Phi(s)$ is the objective function to be minimized, and $s=\left[x_{1}, x_{2}, \cdots, x_{k}\right]^{\mathrm{T}}$ is defined as

$$
s \in \Psi^{k} \text { and } \Psi^{k}=\left\{s \mid a_{j} \leq x_{j} \leq b_{j}\right\}, j=1,2, \ldots, k .
$$

where $a_{j}$ and $b_{j}$ are the boundary values of the $j^{\text {th }}$ element (or $x_{j}$ ) of $s$, and $k$ represents the dimension of the problem or the number of variables. The basic process of the CTS method, which is organized around a simple version of the tabu search, can be summarized as follows.

1. Generate a random point $s$ that belongs to the space $\Psi^{k}$ as the current solution.

2. A set of neighbors, $s^{\prime} \in \Psi^{k}$, is then generated by applying $s$ with a series of perturbations or "moves". Generation of neighbors is defined as follows. The neighborhood space $\Psi^{k}$ of the current solution, $s$, is deemed as a ball $B(s, r)$ centered on $s$ with a radius $r$. Considering a set of concentric balls with radii $h_{0}, h_{1}, \ldots, h_{n}$, the space is partitioned into $n$ concentric 'crowns'. Hence $n$ neighbors of $s$ are obtained by selecting one point randomly inside each crown and eliminating those neighbors that belong to the "tabu list". Figure 6 shows an example that demonstrates the generation of neighbors for a problem with two variables $x_{1}$ and $x_{2}(k=2)$, where the space is partitioned into four $(n=4)$ concentric "crowns", and four neighbors are produced randomly in their own crown areas.

3. Evaluate these neighbors with the objective function, choose the best neighbor $s^{*}$ and replace the starting point $s$ with $s^{*}$ even if it is worse than the current solution. Then update the "tabu list".

4. Clear the "tabu list": in particular those solutions that belong to the "tabu list" can release their tabu status if their "aspiration levels" are sufficiently high.

5. Check the stopping condition and return to step 2 if the condition is not met. Otherwise, stop the iteration procedure and report the results.

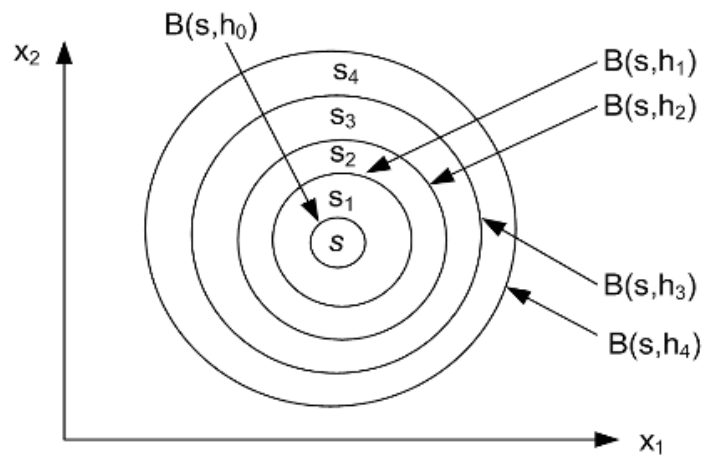

Fig. 6. Partition of the current solution neighborhood, where $x_{1}$ and $x_{2}$ are two variables ( $k$ $=2)$ and $n=4$ concentric "crowns". The neighborhood $s_{j}(j=1,2,3,4)$ is selected randomly in its own crown area (Siarry \& Berthiau, 1997). 
Figure 7 shows the flow chart of the CTS algorithm, where the main stages include the initial solution, generation of neighbors, selection of the solution and tabu list clearance. The strategy of generating neighbors in CTS is more efficient than a naive candidate-list strategy based solely on random sampling, and usually produces neighbors distributed over the whole solution space. Although the method is effective for optimizing functions with two or three variables, its efficiency decreases with an increase in the number of variables of a function such as the case for high-dimension problems. These difficulties can be overcome using a staged continuous Tabu search (SCTS) algorithm which is described next.

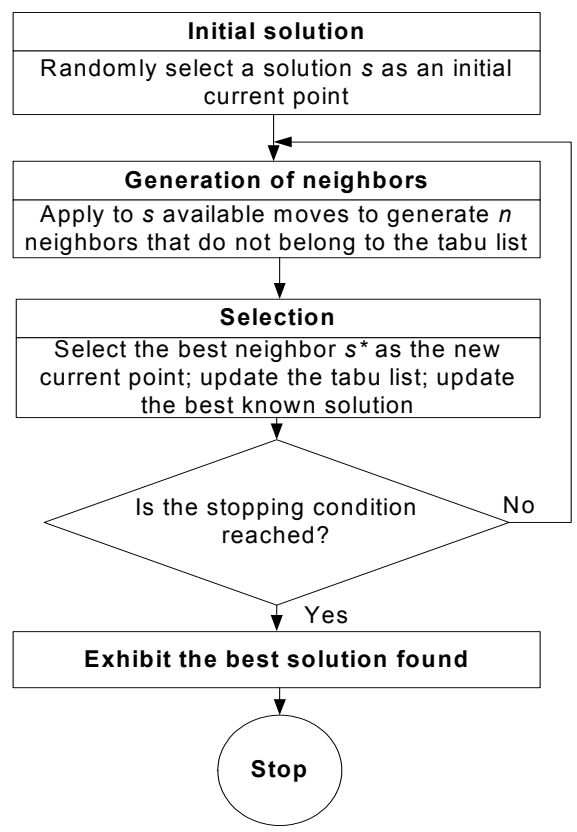

Fig. 7. General flow chart of a continuous Tabu search (CTS) algorithm.

\subsection{Staged continuous Tabu search algorithm}

The staged continuous Tabu search (SCTS) algorithm employs the same rudimentary form of tabu search embodies in the CTS algorithm (Zheng et al., 2005). However, SCTS provides an enhanced candidate-list strategy that subdivides the CTS approach into three independent processes that generate candidate neighbors in a different way (see Fig. 8). The first stage attempts to survey the whole solution space to localize a "prospective point", which is a solution likely to produce a global optimum. The objective of the second stage is to find a point close to the global optimum. The third stage starts from the solution found in the second stage, and eventually converges to the global optimum point. The SCTS algorithm is described below with reference to Fig. 8 which shows the steps of the algorithm. The following notations are used in Fig. 8: $\Psi^{k}$ : space of feasible solutions ( $k$ dimensions); $s_{0}$ : current solution; $n_{1}$ : length of neighbors generated in the first stage (which 
is equal to $k$ here); $n_{2}$ : section number divided within the boundary of every element of $s_{0}$; $n_{3}$ : length of neighbors generated in the third stage; $s^{\prime}$ : the neighborhood of $s_{0} ; s^{*}$ : the best solution in $s^{\prime}$; $s_{\text {opt }}$ : current best solution found; and $M v(j)$ : maximum number of iterations without improvement of $s_{\text {opt }}$ in the $j^{\text {th }}$ stage.

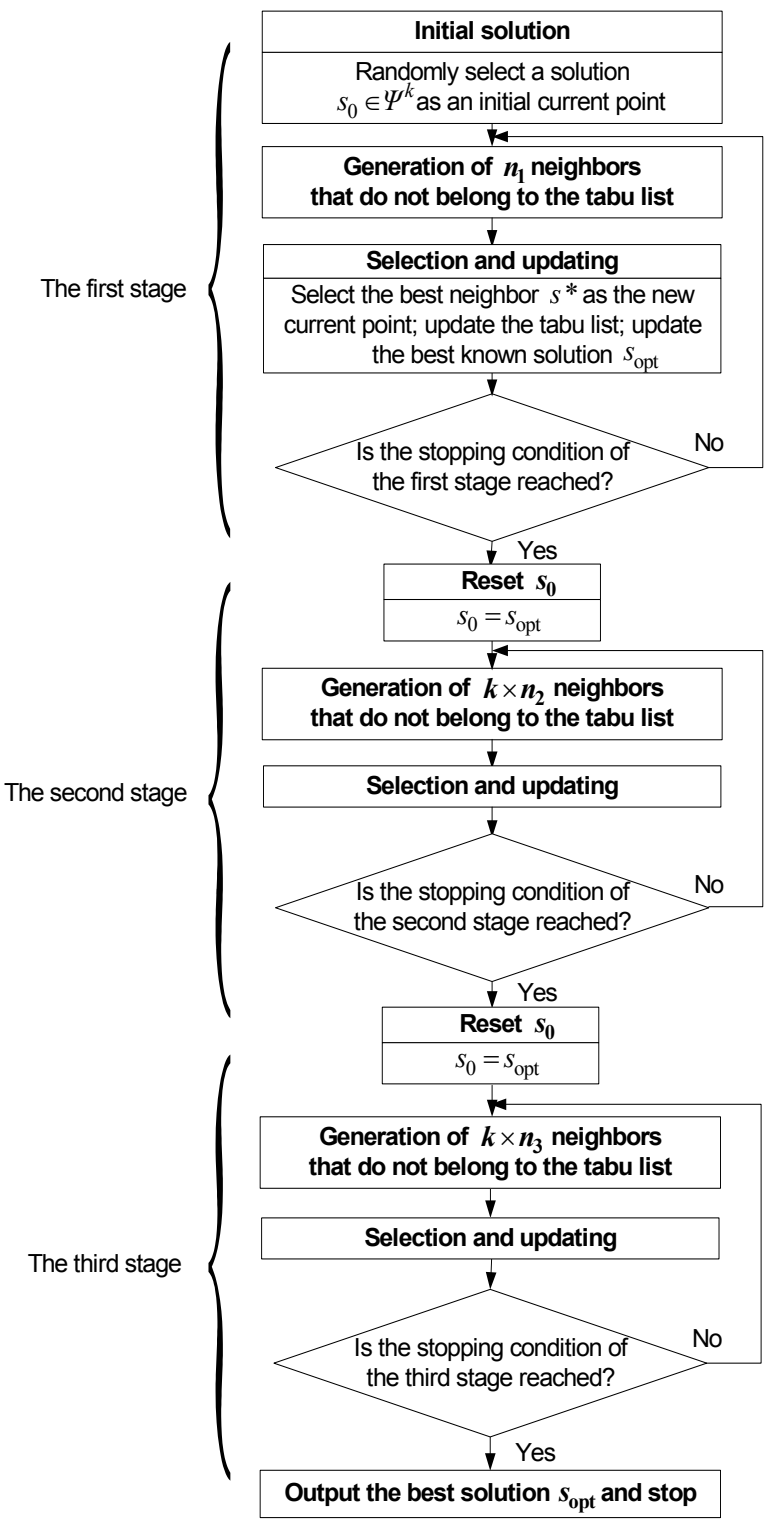

Fig. 8. Algorithmic description of the SCTS algorithm. 
Generation of Neighborhoods: Neighborhoods are generated in a ball $B(s, r)$ centered on $s$ with a radius $r$ (see Fig. 6). All neighbors $s^{\prime}$ meet the condition: $\left|s^{\prime}-s\right| \leq r$. In the first stage, the radius $r_{1}$ is defined so that the ball $B_{1}\left(s, r_{1}\right)$ contains the whole $k$-dimension space $\Psi^{k}$. With radii $r_{1}^{1}, r_{1}^{2}, \cdots, r_{1}^{n_{1}}$, the ball is partitioned into $k$ concentric "crowns" centered on the current solution. One neighbor is produced in each crown. Thus the $j^{\text {th }}$ neighbor, $s_{j}{ }^{\prime}$, is generated with the condition

$$
r_{1}^{j-1} \leq\left|s_{j}{ }^{\prime}-s\right| \leq r_{1}^{j}, \quad\left(r_{1}^{0}=0\right) .
$$

As the ball $B_{1}\left(s, r_{1}\right)$ includes the whole space $\Psi^{k}$, it should be possible for all solutions within it to become neighbors of the current solution, $s$, so that the process can investigate the whole solution space. We define the "moves" to generate neighbors such that some elements of the current solution are randomly replaced. The number of replaced elements depends on different crowns. For example, the $j^{\text {th }}$ neighbor, $s_{j}{ }^{\prime}$, is generated by replacing any $j$ element of the current solution. The radius $r_{2}$ for the generation of neighbors in the second stage is defined as the minimum radius of radii $r_{1}^{1}, r_{1}^{2}, \cdots, r_{1}^{n_{1}}$ defined in the first stage. Followed with another partition process with a set of radii $r_{2}^{1}, r_{2}^{2}, \cdots, r_{2}^{n_{2}}$, the ball $B_{2}\left(s, r_{2}\right)$ is divided into $n_{2}$ sections. The $j^{\text {th }}$ neighbor, $s_{j}{ }^{\prime}$, is generated with a condition given by

$$
r_{2}^{j-1} \leq\left|s_{j}{ }^{\prime}-s\right| \leq r_{2}^{j}, \quad\left(r_{2}^{0}=0\right) .
$$

The minimum radius defined in the first stage is propagated in only one dimension of the current solution. Considering the condition defined in Eq. (23), the boundary can be proportionally divided for every dimension into $n_{2}$ partitions. The neighbors can then be generated by replacing the $j^{\text {th }}$ element of the current solution, $x_{j}{ }^{\prime}$, with a number computed by:

$$
x_{j}{ }^{\prime}=a_{j}+\left(j_{2}+\mu\right) \times \frac{b_{j}-a_{j}}{n_{2}}, \quad \text { where } j=1,2, \ldots, k ; j_{2}=1,2, \ldots, n_{2} ;
$$

where $\mu$ is a random value between 0 and $1, a_{j}$ and $b_{j}$ are defined in Eq. (23), and $k \times n_{2}$ is the number of neighbors in the stage. The minimum of radii $r_{2}^{1}, r_{2}^{2}, \cdots, r_{2}^{n_{2}}$ is set as radius $r_{3}$ to generate neighbors in the third stage. Instead of partitioning to generate neighbors, the radius of the ball $B_{3}\left(s, r_{3}\right)$ decreases with an increase in the iteration number. The generation of the $j^{\text {th }}$ neighbor is defined as

$$
x_{j}^{\prime}=x_{j}+\mu_{j} \times \frac{b_{j}-a_{j}}{n_{2}} \times \frac{M_{3}-m}{M_{3}}
$$


where $x_{j}$ and $x_{j}{ }^{\prime}$ are the $j^{\text {th }}$ elements of the current solution $s$ and the neighbor $s^{\prime}$ produced, respectively, $m$ is the iteration number without any improvement on the current solution, and $M_{3}$ is the maximum allowable number of iterations without any improvement on the current solution. As pointed out previously, the neighbors in the first stage are generated in the largest possible range so as to explore most of the space. While in the last stage, only a reduced space is used so that the solution will eventually converge to the global optimum.

Tabu list: In the underlying TS algorithm, a tabu list stores some solutions that have recently been selected. It is used to qualify the algorithm to select solutions that have not been selected before so as to escape from being recycled. Because the three stages in the SCTS algorithm are independent of each other, the tabu lists in these stages are thus independent of each other. The list obtained in the first stage will store those 'prospective solutions' found in recent iterations. In the second and third stages, the lists will store the attributes of 'moves' or perturbations that generate the best neighbors in recent iterations. The tabu list in each stage is always reset at the beginning of each stage.

Stopping conditions: The stopping conditions for the three processes are defined below.

1. The program will stop after a given number of iterations when there is no improvement on the value of the objective function. The number of iterations varies in different stages.

2. The result satisfies the prescribed condition. An example of the prescribed condition is the known global optimum of a benchmark test function.

3. The search procedure will stop after it has completed a pre-defined maximum number of iterations.

All stages will be terminated if any one of these stopping conditions is satisfied. That is, if the process is in the first or second stage, it will move into the next stage. However, if the process is in the third stage, the algorithm will stop and report the result.

The sensitivities of several main parameters of the CTS algorithm were discussed (Siarry \& Berthiau, 1997). Usually, these parameters should be adjusted empirically according to the nature of the problem so as to achieve an efficient optimization. As the SCTS algorithm is derived from the CTS algorithm, it is found that the properties of some parameters in the SCTS algorithm are similar to those of the CTS algorithm. These parameters are not analyzed individually, and instead a set of empirical values is applied in the experiments for testing the benchmark functions. These empirical values are listed in Table 1.

\begin{tabular}{|c|c|}
\hline Parameters used in the SCTS algorithm & $\begin{array}{c}\text { Parameters used in the } \\
\text { benchmark functions }\end{array}$ \\
\hline Number of neighbors in the first stage $\left(n_{1}\right)$ & Number of variables \\
\hline Number of sections in the second stage $\left(n_{2}\right)$ & 5 \\
\hline Number of neighbors in the third stage $\left(n_{3}\right)$ & Number of variables \\
\hline Number of neighbors in the second stage & $5 \times$ number of variables \\
\hline $\begin{array}{c}\text { Maximum number of iterations without any } \\
\text { improvement on the objective function value }(M v)\end{array}$ & $\left\{1^{\text {st }}\right.$ stage, $2^{\text {nd }}$ stage, $3^{\text {rd }}$ stage $\}$ \\
\hline Maximum number of iterations of the SCTS algorithm & 8000 \\
\hline
\end{tabular}

Table 1. Parameters of the SCTS algorithm used for testing the benchmark functions. 
Numerical experimental results: To demonstrate the effectiveness of the SCTS algorithm, the important parameters to be studied are convergence, speed and robustness. Convergence refers to the evaluation of the search for the global optimum of a function. The test for convergence employed here is the relative error between the optimum obtained by the algorithm, $X_{\mathrm{opt}}$, and a theoretical (or known) value of the optimum, $X_{\text {theo }}$, of the function. The relative error, $E_{\text {relative }}$, is defined as (Andre et al., 2000)

$$
E_{\text {relative }}=\frac{\left|X_{\text {opt }}-X_{\text {theo }}\right|}{X_{\text {theo }}}
$$

If $X_{\text {theo }}=0$, Eq. (28) is reduced to

$$
E_{\text {relative }}=\left|X_{\text {opt }}-X_{\text {theo }}\right|
$$

The criterion of speed refers to the time taken by the algorithm to find the global optimum of an objective function. As the computation time also depends on the speed of the computer, it is better to define the speed criterion by determining the number of evaluations of the objective function required till a global optimum is found. Robustness means that the algorithm is versatile and can be applied to solving a variety of functions. A set of commonly used benchmark functions with known global optima (as listed in the Appendix) is employed to test the algorithm. These benchmark test functions represent various practical problems in engineering. To obtain a statistical comparison of the optimization results, every test is performed 100 times (starting from various randomly selected points) to ensure that the results obtained are reliable.

Table 2 shows the results obtained from the CTS (Siarry \& Berthiau, 1997) and SCTS algorithms for the four test functions with two and three variables. The criterion of success is the percentage of trials (out of 100 tests for each function) required to obtain the global optimum with a relative error of $<1 \%$. From the table, both algorithms can successfully find the global optima of all the four test functions which are given in the Appendix. However, the number of evaluations of the Goldprice and Shubert test functions required by the SCTS algorithm (i.e., 696 and 521, respectively) is much smaller than those required by the CTS algorithm (i.e., 1636 and 1123, respectively). This means that the SCTS algorithm has a faster computation rate than that of the CTS algorithm for these two particular test functions. However, the two algorithms have about the same computation rate for the Hartmann 1 and Branin functions.

\begin{tabular}{|c|c|c|c|c|}
\hline \multirow{2}{*}{ Function } & \multicolumn{2}{|c|}{ Success rate (\%) } & \multicolumn{2}{c|}{$\begin{array}{c}\text { Number of evaluations of } \\
\text { objective or test functions }\end{array}$} \\
\cline { 2 - 5 } & CTS & SCTS & CTS & SCTS \\
\hline Goldprice & 100 & 100 & 1636 & 696 \\
\hline Hartmann1 & 100 & 100 & 528 & 691 \\
\hline Branin & 100 & 100 & 668 & 491 \\
\hline Shubert & 100 & 100 & 1123 & 521 \\
\hline
\end{tabular}

Table 2. Experimental data of the SCTS and CTS algorithms. 
Table 3 shows a comparison of the experimental data of various test functions obtained by the SCTS algorithm and an improved genetic algorithm (IGA). Note that the IGA algorithm can potentially yield a complete set of optima of multimodal problems (Andre, Siarry \& Dognon, 2000). These test functions have variables ranging from 1 to 20 as given in the Appendix. In the table, Max and Min are, respectively, the maximum and minimum values of a set of optima found over 100 tests. The SCTS algorithm outperforms the IGA algorithm in two ways. The SCTS algorithm has one advantage in that it can find the global optima of the test functions (i.e., Brown1, Brown 3 and F10n functions) with $100 \%$ success rate and with very low relative errors; while the IGA algorithm has very low success rates and very high relative errors of these test functions. The other advantage of the SCTS algorithm over the IGA algorithm is that it has a much smaller computation time due to the smaller number of evaluations of the test functions. In addition, for $100 \%$ success rates of the test functions achieved by both algorithms, the SCTS algorithm has much smaller relative errors than those of the IGA algorithm. It can thus be concluded from the results shown in Tables 2 and 3 that the SCTS algorithm outperforms the CTS algorithm and the IGA algorithm in terms of higher success rate and higher computation efficiency. Next section describes the use of the SCTS algorithm for the development of a hybrid Tabu search algorithm which is a hybrid of the global SCTS algorithm and the local Quasi-Newton algorithm.

\section{Hybrid Tabu search algorithm for optimization and fabrication of non- uniform fiber Bragg gratings}

\subsection{Staged continuous Tabu search algorithm for optimization of non-uniform fiber Bragg gratings}

This section describes the SCTS algorithm (as described in Section 3.3) for use in the optimization of non-uniform FBGs, and it is schematically shown in Fig. 9.

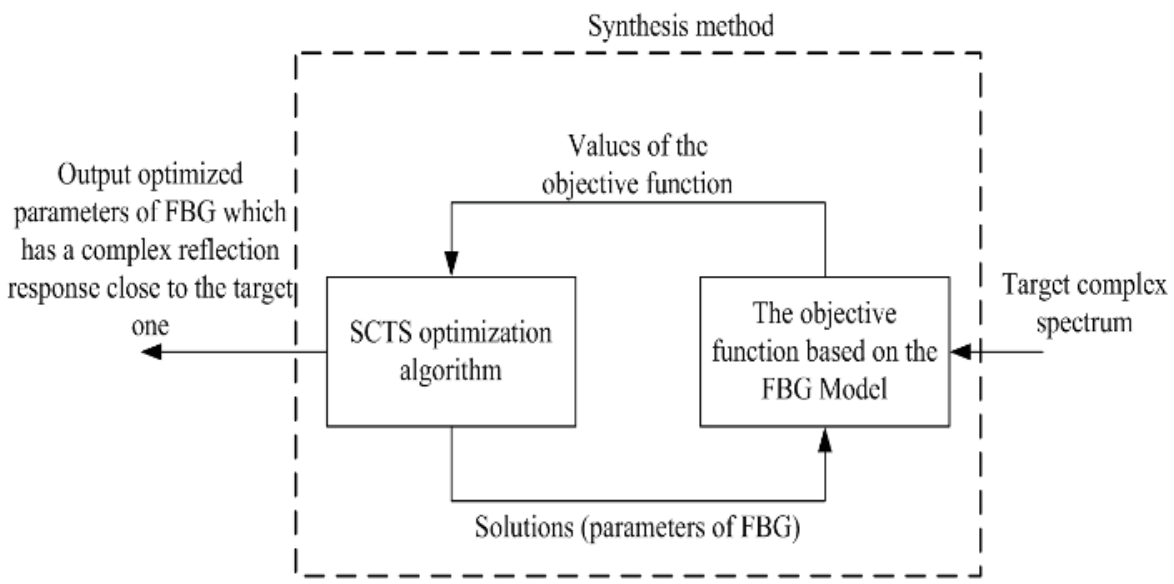

Fig. 9. Block diagram of the SCTS algorithm for use in the optimization of FBGs.

The synthesis problem of an FBG is formulated as an objective function which measures the error between the designed reflection frequency response of the FBG using the SCTS algorithm and the target frequency response. The synthesized FBG using the SCTS algorithm can be carried out using the steps as described below. 


\begin{tabular}{|c|c|c|c|c|c|c|c|c|}
\hline \multirow[t]{2}{*}{ Function } & \multirow{2}{*}{$\begin{array}{l}\text { Number } \\
\text { of } \\
\text { variables }\end{array}$} & \multirow{2}{*}{$\begin{array}{c}\text { Theoretical } \\
\text { minimum } \\
\text { value of } \\
\text { optimum }\end{array}$} & \multicolumn{2}{|c|}{$\begin{array}{c}\text { Minimum value of a set } \\
\text { of optima found } \\
\text { (Min) }\end{array}$} & \multicolumn{2}{|c|}{$\begin{array}{l}\text { Maximum value of a } \\
\text { set of optima found } \\
\text { (Max) }\end{array}$} & \multicolumn{2}{|c|}{$\begin{array}{c}\text { Average of } \\
\text { relative errors } \\
\text { over } 100 \text { tests }(\%\end{array}$} \\
\hline & & & IGA & SCTS & IGA & SCTS & IGA & SCTS \\
\hline $\mathrm{F} 1$ & 1 & -1.1232 & -1.1232 & -1.1232 & -1.1139 & -1.1223 & 0.03 & 0 \\
\hline F3 & 1 & -12.0312 & -12.0312 & -12.0312 & -11.9270 & -12.0203 & 0.12 & 0 \\
\hline Branin & 2 & 0.3979 & 0.3979 & 0.3979 & 0.4018 & 0.3983 & 0.48 & 0 \\
\hline Goldprice & 2 & 3 & 3.003 & 3.002 & 3.0296 & 3.0029 & 0.43 & 0 \\
\hline Shubert1 & 2 & -186.7309 & -186.6857 & -186.7304 & -184.9554 & -186.3406 & 0.53 & 0.014 \\
\hline Shubert2 & 2 & -186.7309 & -186.7047 & -186.7302 & -184.9295 & -185.9505 & 0.53 & 0.024 \\
\hline Shubert & 2 & -186.7309 & -186.7280 & -186.7269 & -184.8753 & -186.5490 & 0.49 & 0.0003 \\
\hline Hartmann1 & 3 & -3.8628 & -3.8611 & -3.8621 & -3.8246 & -3.8591 & 0.51 & 0.018 \\
\hline Brown1 & 20 & 2 & 8.5516 & 2.0018 & 111.2914 & 2.0020 & 2692.67 & 0.05 \\
\hline Brown3 & 20 & 0 & 0.6746 & 0.0006 & 5.9122 & 0.0010 & 2.324 & 0.0009 \\
\hline F5n & 20 & 0 & 0.0022 & 0.0001 & 0.5906 & 0.0010 & 0.067 & 0.0004 \\
\hline F10n & 20 & 0 & 0.0496 & 0.0001 & 4.0660 & 0.0010 & 1.197 & 0.0008 \\
\hline F15n & 20 & 0 & 0.0034 & 0.0003 & 0.7361 & 0.0009 & 0.075 & 0.0008 \\
\hline
\end{tabular}

Table 3. Experimental data of the test functions obtained by the SCTS algorithm and the IGA a 
1. When the target frequency response (or complex spectrum) is input into the synthesis method, the FBG model is formulated as an objective function to be minimized. The FBG model can either be based on the cascade of serially-connected uniform subgratings (Section 2.2.1) for the synthesis of the desired magnitude response (where the phase response is not important) or the cascade of serially-connected apodized subgratings (Section 2.2.2) for the synthesis of the desired frequency response (which includes both the magnitude and phase responses).

2. The algorithm will produce a set of solutions. These solutions include the index modulation profiles, $\Delta n_{\mathrm{ac}, j}(j=1,2, \cdots M)$, of the cascade of uniform sub-gratings (Eq.

(5)) or the cascade of the apodized sub-gratings (Eq. (17)).

3. These solutions are sent to the objective function built in step (1). The values of the objective function are calculated and sent back to the algorithm.

4. The algorithm checks the calculated values of the objective function. If the pre-defined stopping conditions are not reached, the process will enter the next iteration and will be recycled from step (2). Otherwise, the algorithm will output the best solution found, that is, the optimized structural parameters $\left(\delta l_{j}, \Delta n_{\mathrm{ac}, j}, f_{j}, \Lambda_{j}\right.$ and $\left.\Delta n_{\mathrm{dc}, j}\right)$ of the designed FBG.

The SCTS algorithm has been used for the synthesis of FBG-based bandpass filters using the non-uniform FBG model based on the cascade of serially-connected uniform sub-gratings (Zheng et al., 2005).

\subsection{Hybrid Tabu algorithm for optimization of non-uniform fiber Bragg gratings}

The global SCTS algorithm (Section 4.1) generally cannot produce an optimum solution of the synthesized FBG, which may make practical realization difficult. Unfortunately, local optimization methods such as the Quasi-Newton method also cannot yield an optimum solution of the FBG synthesis problem due to the multimodal and ill-conditioned character of the nonlinear objective function. To improve the convergence of the global optimization algorithm, a hybrid algorithm combining the global optimization algorithm and the local optimization algorithm is a better approach for solving the nonlinear objective functions. The motivation of this section is to thus present a hybrid Tabu algorithm for the synthesis and fabrication of FBG-based bandpass and linear phase filters (Ngo, Zheng, Ng, Tjin \& Binh, 2007). The hybrid Tabu algorithm (see Fig. 10) is a two-tier search that employs the global SCTS algorithm (Section 4.1) and a local optimization algorithm (i.e., the QuasiNewton method which has high efficiency in solving multimodal nonlinear optimization problems (Shanno, 1970)).

In Fig. 10, the first step of the global SCTS algorithm produces a "promising" grating structure, which can either consist of the cascade of serially-connected uniform sub-gratings (Section 2.2.1) or apodized sub-gratings (Section 2.2.2), with the optimized structural parameters $\left(\delta l_{j}, \Delta n_{\mathrm{ac}, j}, f_{j}, \Lambda_{j}\right.$ and $\left.\Delta n_{\mathrm{dc}, j}\right)$. In this first step, the length of the "promising" index modulation profile is divided into $N$ equally-spaced points, that is, $\Delta n_{\mathrm{ac}, j}(j=1,2, \cdots N)$ is sampled at the discrete point $z_{j}$, where $\Delta n_{\mathrm{ac}, j}$ can be assumed to be constant for each sub-grating section. The index modulation profile, $\Delta n_{\mathrm{ac}, j}(j=1,2, \cdots N)$, of this "promising" grating structure is further optimized by the local Quasi-Newton algorithm. In this second step, the length of the "promising" index modulation profile is 
divided into $M$ equally-spaced points, that is, $\Delta n_{\mathrm{ac}, j}(j=1,2, \cdots M)$ is sampled at the discrete point $z_{j}$, where $\Delta n_{\mathrm{ac}, j}$ can be assumed to be constant for each sub-grating section. The TMM method described in Section 2 can be used to calculate the frequency responses of the designed FBG-based filters. To demonstrate its effectiveness, the hybrid Tabu algorithm is applied to the design and fabrication of FBG-based bandpass filters and the design of FBGbased linear phase filters, and this is described below.

Hybrid optimization algorithm

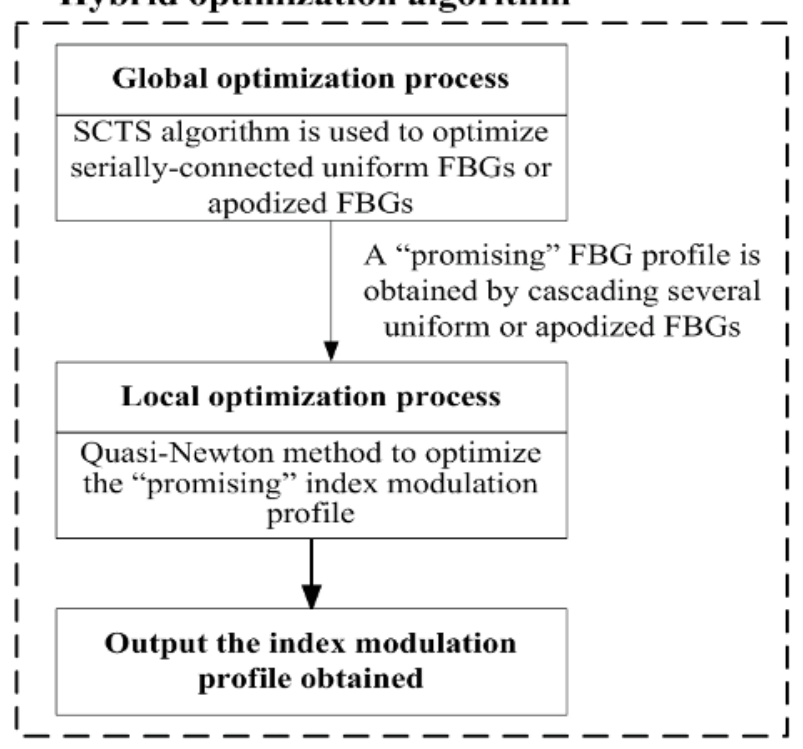

Fig. 10. Schematic diagram of the hybrid Tabu algorithm for use in the optimization of FBGs.

\subsection{Design and fabrication of FBG-based bandpass filters}

Bandpass optical filters are attractive for performing many signal processing and filtering functions in optical sensors and optical communications. This section describes the use of the hybrid Tabu algorithm described in Section 4.2 for the synthesis and fabrication of bandpass optical filters. As only the magnitude response (but not the phase response) of the bandpass filter is to be synthesized, the FBG model based on the cascade of seriallyconnected uniform (instead of apodized) sub-gratings (section 2.2.1) is employed here. The magnitude response of the FBG-based bandpass filter to be synthesized is a function of the index modulation profile, $\overrightarrow{\mathrm{Var}}$, and is given by

$$
R_{j}(\overrightarrow{\mathrm{Var}}) ; \quad j \in \text { wavelength window }
$$

where $\overrightarrow{\mathrm{Var}}=\left[\Delta n_{\mathrm{ac}, 1}, \Delta n_{\mathrm{ac}, 2}, \cdots, \Delta n_{\mathrm{ac}, M}\right]$. The target magnitude response of the bandpass filter with a bandwidth of $25 \mathrm{GHz}$ (or $0.2 \mathrm{~nm}$ in the $1550 \mathrm{~nm}$ wavelength window) is given by 


$$
R_{\text {target }, j}=\left\{\begin{array}{rr}
1 ; & 1549.9 \mathrm{~nm} \leq \lambda_{j} \leq 1550.1 \mathrm{~nm} \\
0 ; & \lambda_{j}<1549.9 \mathrm{~nm} \text { and } \lambda_{j}>1550.1 \mathrm{~nm}
\end{array}\right.
$$

The step size or wavelength resolution of $0.01 \mathrm{~nm}$ is used in the optimization. The objective function to be optimized is a measure of the error between the calculated magnitude response of the optimized FBG (Eq. (30)) and the target magnitude response (Eq. (31)) and is given by

$$
\operatorname{error}\left(\overrightarrow{\Delta n_{\mathrm{ac}}}\right)=\sum_{\substack{j \in \text { wavelength } \\ \text { window }}} W_{j}^{R} \times \sqrt{\left|R_{j}(\overrightarrow{\mathrm{Var}})-R_{\text {target }, j}\right|}
$$

where $R_{j}(\overrightarrow{\mathrm{Var}})$ is the reflectivity of the FBG's magnitude response at the $j^{\text {th }}$ wavelength ( $\left.\lambda_{j}\right)$ which is computed using the hybrid Tabu algorithm and Eq. (14) and $R_{\text {target, } j}$ is the reflectivity of the target magnitude response at the $j^{\text {th }}$ wavelength which is defined in Eq. (31). The weight parameter at the $j^{\text {th }}$ wavelength is given by

$$
W_{j}^{R}= \begin{cases}10 ; & \lambda_{j} \in \text { stopband } \\ 1 ; & \lambda_{j} \in \text { passband }\end{cases}
$$

where the weight values in the stopband are chosen to be 10 (ten) times more than those in the passpand in order to achieve effective suppression of the sidelobes. An additional constraint can be imposed on $\overrightarrow{\Delta n_{\mathrm{ac}}}=\left[\Delta n_{\mathrm{ac}, 1}, \Delta n_{\mathrm{ac}, 2}, \cdots, \Delta n_{\mathrm{ac}, M}\right]$ by setting appropriate values of $\Delta n_{\mathrm{ac}, 1}$ and $\Delta n_{\mathrm{ac}, M}$ to suit the condition(s) of a particular fabrication system. In this design, $M=40$ and $\overrightarrow{\Delta n_{\mathrm{ac}}}=[0,0.0002]$ are chosen, and the chosen parameter values of the SCTS algorithm are listed in Table 1 . The beam size of our frequency-doubled argon laser system is $\sim 0.5 \mathrm{~mm}$ and thus the sub-grating length, $\delta l_{j}$, (which must be at least equal to the beam size) is chosen to be $\sim 0.5 \mathrm{~mm}$ to meet the fabrication requirement. The hybrid Tabu algorithm is to find the global optimum of Eq. (32). The optimized bandpass filter using the hybrid Tabu algorithm has 40 sub-grating sections and thus a total grating length of $L_{g}=20 \mathrm{~mm}$. Figure 11(a) shows the optimized index modulation profile, $\overrightarrow{\Delta n_{\mathrm{ac}}}$, of the FBGbased bandpass filter based on the hybrid Tabu algorithm. For comparison, the solid line of Fig. 11(b) shows the index modulation profile obtained from the first stage of the hybrid Tabu method (i.e., the SCTS process, see Fig. 10), and this profile is used as the "promising" structure in the second stage of the hybrid method. The dashed line of Fig. 11(b) shows the index modulation profile of a standard sine-apodized FBG-based bandpass filter (without using optimization) (Kashyap, 1999). The corresponding reflection magnitude responses of these three index modulation profiles are shown in Fig. 12. The SCTS-optimized bandpass filter outperforms the standard apodized bandpass filter (without using optimization) in terms of sharper roll-offs and larger sidelobe suppression levels, demonstrating the 
advantage of employing optimization. The hybrid-optimized bandpass filter using the hybrid Tabu algorithm performs best because the roll-offs on the edges of the bandpass response are steepest and the sidelobes have the greatest suppression levels of less than -30 dB. The hybrid-optimized filter with results shown in Fig. 11(a) and Fig. 12 can be fabricated using the fabrication method reported by (Asseh, Storoy, Sahlgren, Sandgren \& Stubbe, 1997; Ngo, Zheng, Ng, Tjin \& Binh, 2007), and this is described as follows. The 40 subgratings in a $20-\mathrm{mm}$ long grating were exposed in sequence using UV pulses. Each subgrating has a few hundred periods. The index-modulation depth of each sub-grating can be tuned to the designed value (as shown in Fig. 11(a)) by adjusting the offset of the fiber dithering away from the phase mask. That is, if the offset of the fiber dithering is half of the sub-grating period, the index modulation will be completely averaged out (i.e., no index modulation). However, if there is no offset of the fiber dithering from the phase mask, the index modulation value will be largest. In Fig. 13, the measured reflection magnitude response of the fabricated 20-mm long hybrid-optimized FBG-based bandpass filter using this fabrication method (the solid line) has steeper roll-offs than those of the fabricated 20mm long standard (or non-optimized) uniform FBG-based bandpass filter (the dashed line). The figure shows that the fabricated filter has a sidelobe suppression level of $-20 \mathrm{~dB}$ which is sufficient for many practical applications. This suppression level is greater than the designed $-30 \mathrm{~dB}$ level (see Fig. 12) due probably to fabrication errors such as the positioning error of the translation stage, the fluctuation of the UV laser power and tiny dirty spots on the phase mask.

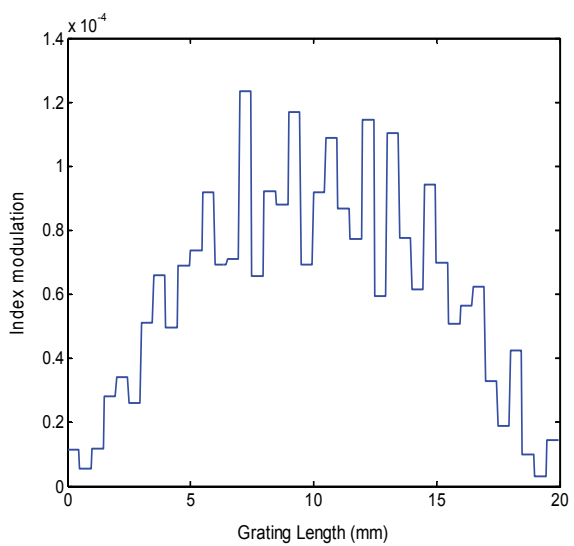

(a)

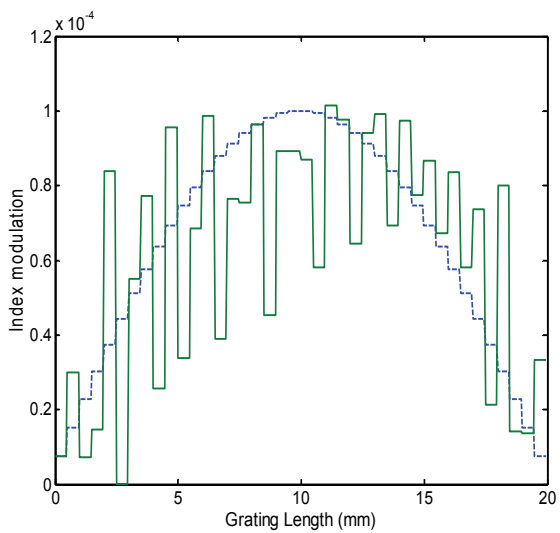

(b)

Fig. 11. (a) The optimized index modulation profile, $\overrightarrow{\Delta n_{\mathrm{ac}}}$, of the FBG-based bandpass filter based on the hybrid Tabu algorithm. (b) The solid line shows the index modulation profile obtained from the first stage of the hybrid Tabu method. The dashed line shows the index modulation profile of a standard sine-apodized FBG-based bandpass filter (without using optimization). 


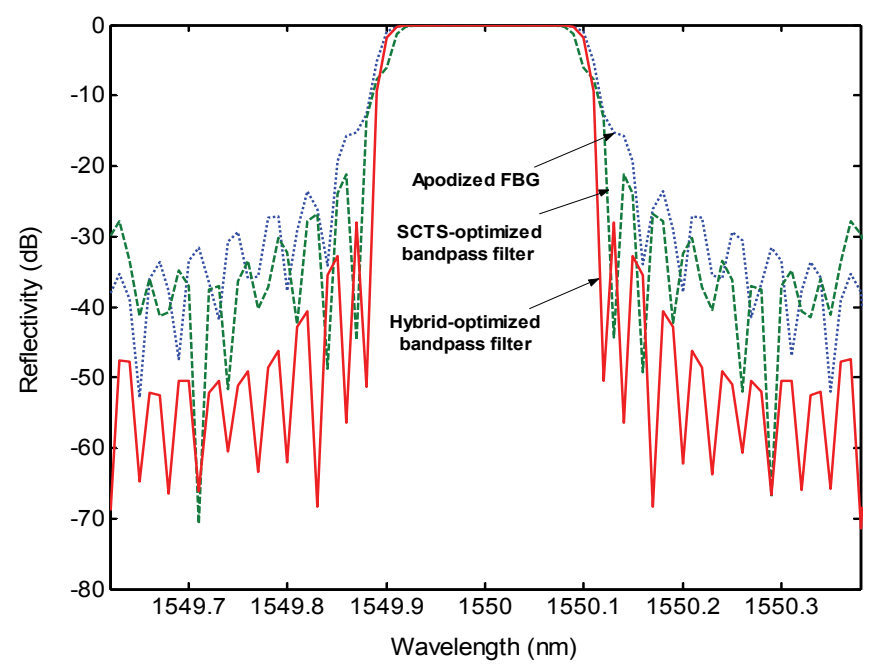

Fig. 12. Simulation results of the reflection magnitude responses of the three index modulation profiles shown in Fig. 11.

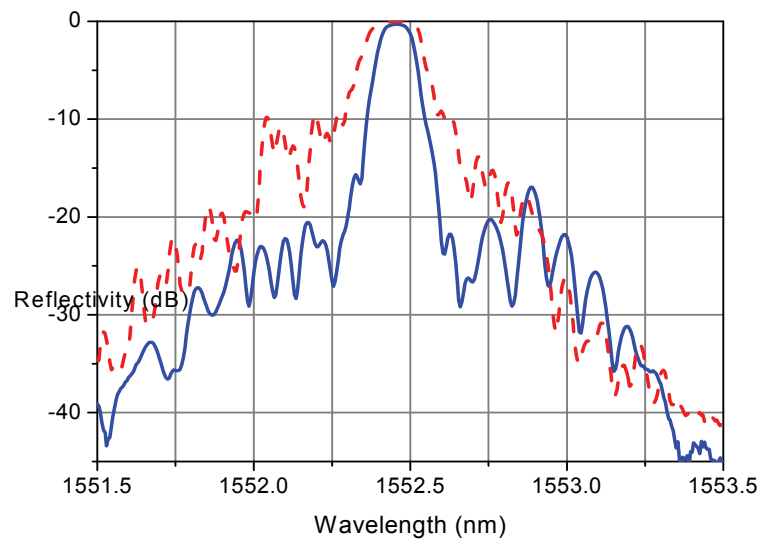

Fig. 13. Measured reflection magnitude responses of a 20-mm long hybrid-optimized FBGbased bandpass filter (solid line) and a standard 20-mm long uniform FBG-based bandpass filter (without using optimization) (dashed line).

\subsection{Design of FBG-based linear phase filters}

Linear phase optical filters with near-squared magnitude responses are attractive for signal processing and filtering in optical sensors and optical communications systems while maintaining the essential features of the signals. One important application of such a filter is a high-speed (> $10 \mathrm{Gbit} / \mathrm{s}$ ) add/drop multiplexer that can add or drop a particular signal wavelength without introducing distortion to the signal. To further demonstrate the 
effectiveness of the hybrid Tabu method, this section presents the design of three FBG-based linear phase filters with different grating lengths and with $50 \mathrm{GHz}$ bandwidth each (i.e., 0.4 $\mathrm{nm}$ in the 1550-nm wavelength window). Both the magnitude and phase responses of the linear phase filters are to be synthesized using the hybrid Tabu algorithm. Two stages are involved in the hybrid Tabu algorithm and they are described below. The first stage of the hybrid Tabu algorithm employs the non-uniform FBG model based on the cascade of serially-connected apodized sub-gratings (Section 2.2.2). The second stage of the hybrid Tabu algorithm employs the non-uniform FBG model based on the cascade of serially-connected uniform sub-gratings (Section 2.2.1) instead of apodized sub-gratings (Section 2.2.2) because it is much simpler to fabricate the former than the later.

First stage of the hybrid Tabu algorithm: The first stage of the hybrid Tabu algorithm is the global SCTS algorithm (see Fig. 10). The desired magnitude response of a FBG-based linear phase filter with a bandwidth of $0.4 \mathrm{~nm}$ (or $50 \mathrm{GHz}$ in the $1550 \mathrm{~nm}$ wavelength window) to be synthesized is a function of the index modulation profile, $\overrightarrow{\mathrm{Var}}$, and is given by

$$
R_{j}(\overrightarrow{\mathrm{Var}}) ; j \in \text { wavelength window }
$$

The row vector $\overrightarrow{\mathrm{Var}}$ is given by

$$
\overrightarrow{\mathrm{Var}}=\left[\Delta n_{\mathrm{ac}, 1}^{\max }, \cdots, \Delta n_{\mathrm{ac}, N}^{\max }, \delta l_{1}, \cdots, \delta l_{N}\right]
$$

where $\Delta n_{\mathrm{ac}, j}^{\max }$ and $\delta l_{j}$ are the maximum amplitude of the index modulation and the subgrating length of the $j$ th sub-grating, respectively, that are to be optimized. The design objective is to find $\overrightarrow{\mathrm{Var}}$ that produces the optimized magnitude and linear phase responses as close as possible to the target ones. The parameters that are not optimized are $\Lambda_{j}$ and $\Delta n_{\mathrm{dc}, j}(z)=\Delta n_{\mathrm{dc}, j}$ (and hence $\bar{n}_{\mathrm{eff}, j}$ according to Eq. (2)), which are the period and the "dc" index change, respectively, and they are fixed or constant values for ease of fabrication. The apodization function used is the quadratic-sine apodization profile which is defined in Eq. (21) as $f_{j}=\sin ^{2}\left(\pi z / \delta l_{j}\right)$. Compared to other apodization profiles as defined in Eqs. (18)-(20), the quadratic-sine apodization profile can provide a larger sidelobe suppression level and a higher reflectivity in the passband of the magnitude response. The step size or wavelength resolution of $0.01 \mathrm{~nm}$ is used in the optimization. The target magnitude response of a linear phase filter (with $0.4 \mathrm{~nm}$ or $50 \mathrm{GHz}$ bandwidth in the $1550 \mathrm{~nm}$ wavelength window) is given by

$$
R_{\text {target }, j}=\left\{\begin{array}{lr}
1 ; & 1549.8 \mathrm{~nm} \leq \lambda_{j} \leq 1550.2 \mathrm{~nm} \text { (passband) } \\
0 ; & \lambda_{j}<1549.8 \mathrm{~nm} \text { and } \lambda_{j}>1550.2 \mathrm{~nm} \text { (stopband) }
\end{array}\right.
$$

The objective function to be optimized is a measure of the error between the optimized magnitude response of the FBG (Eq. (30)) and the target magnitude response (Eq. (36)) and is given by

$$
\operatorname{error}(\overrightarrow{\mathrm{Var}})=\sum_{j \in \text { window }} W_{j}^{R} \times \sqrt{\left|R_{j}(\overrightarrow{\mathrm{Var}})-R_{\mathrm{target}, j}\right|}+b \times \sum_{k \in \text { passband }}\left|D_{k}(\overrightarrow{\mathrm{Var}})-D_{\text {target }, k}\right|
$$


where $R_{j}(\overrightarrow{\mathrm{Var}})$ is the reflectivity of the FBG's magnitude response at the $j^{\text {th }}$ wavelength $\left(\lambda_{j}\right)$ which is calculated based on the hybrid Tabu algorithm and Eq. (14). In the stopband, suppression of sidelobes in wavelength regions close to the center wavelength is more critical than in other wavelength regions. To achieve this and to increase the optimization efficiency, the weight parameter of the $j^{\text {th }}$ wavelength, $W_{j}^{R}$, for the reflectivity of the magnitude response is given by

$$
W_{j}^{R}=\left\{\begin{array}{l}
\frac{\lambda_{0}}{\left|\lambda_{0}-\lambda_{j}\right|} ; \quad \lambda_{j} \in \text { stopband } \\
\varepsilon ; \quad \lambda_{j} \in \text { passband }
\end{array}\right.
$$

where $\lambda_{0}$ is the center wavelength. The numerical value of $\varepsilon$ was chosen by means of trials and errors by carrying out many simulations, and it was found that $\varepsilon=1$ gave promising results that meet the specifications of the filter designs. However, in general, it is possible that other values of $\varepsilon$ could give satisfying optimization results. $D_{k}(\overrightarrow{\mathrm{Var}})$ is the dispersion at the $k^{\text {th }}$ wavelength which is calculated using the hybrid Tabu algorithm and Eq. (16). The target dispersion at the $k^{\text {th }}$ wavelength of a linear phase filter is given by

$$
D_{\text {target, } k}=0 ; \quad \lambda_{k} \in \text { passband. }
$$

The adjustable parameter $b$ is used to dynamically balance the error between the reflectivity of the magnitude response and the dispersion response. The first stage of the hybrid Tabu algorithm (i.e., the SCTS algorithm) is to find the global optimum of Eq. (37). Additional constraints (e.g., $\Lambda_{j}$ ) can be included in $\overrightarrow{\mathrm{Var}}$ in (Eq. (35) to suit the fabrication condition(s) or limitation(s) of a particular fabrication system to ensure that the designed filter can be practically realized. From Eq. (35), the length of $\overrightarrow{\mathrm{Var}}$ for $N$ cascaded serially-connected apodized sub-gratings is $2 \times N$ because $\overrightarrow{\mathrm{Var}}$ has two variables; thus the filter synthesis problem is an optimization problem with $2 \times N$ variables. Satisfying results can be achieved with $N=5$ while minimizing the computation time. From Eq. (35), elements from the $(N+$ 1)th to the $(2 \times N)$ th of the vector $\overrightarrow{\mathrm{Var}}$ describe the lengths of the apodized sub-gratings. By setting appropriate boundary values for these elements, the total grating length $L_{\mathrm{g}}$ can be controlled in the optimization to make fabrication feasible.

Second stage of the hybrid Tabu algorithm: After a "promising" index modulation profile (see Eq. (35)) is obtained from the first stage of the hybrid Tabu process as described above, it is set as the initial solution for the second stage of the hybrid Tabu algorithm. In the second stage of the hybrid method (i.e., the local Quasi-Newton method), the index modulation profile is divided into $M$ sections and hence $M$ variables in the optimization. The $M$ sections are equally spaced. The index modulation of each section is assumed to be constant. The non-uniform FBG model employed in this second stage is based on the cascade of seriallyconnected uniform sub-gratings (Section 2.2.1) instead of apodized sub-gratings (Section 2.2.2) because it is much easier to fabricate the former than the later. Thus the error function for the index modulation, $\overrightarrow{\Delta n_{\mathrm{ac}}}=\left[\Delta n_{\mathrm{ac}, 1}, \Delta n_{\mathrm{ac}, 2}, \cdots, \Delta n_{\mathrm{ac}, M}\right]$, to be optimized can be defined as 


$$
\text { error }\left(\overrightarrow{\Delta n_{\mathrm{ac}}}\right)=\sum_{j \in \text { window }} W_{j}^{R} \times \sqrt{\left|R_{j}\left(\overrightarrow{\Delta n_{\mathrm{ac}}}\right)-R_{\mathrm{target}, j}\right|}+b \times \sum_{k \in \text { passband }}\left|D_{k}\left(\overrightarrow{\Delta n_{\mathrm{ac}}}\right)-D_{\text {target }, k}\right|
$$

where $R_{j}\left(\overrightarrow{\Delta n_{\mathrm{ac}}}\right)$ is the reflectivity of the FBG's magnitude response at the $j^{\text {th }}$ wavelength $\left(\lambda_{j}\right)$ which is calculated based on the hybrid Tabu algorithm and Eq. (14), $D_{k}\left(\overrightarrow{\Delta n_{\mathrm{ac}}}\right)$ is the dispersion at the $k^{\text {th }}$ wavelength which is calculated using the hybrid Tabu algorithm and Eq. (16), $W_{j}^{R}$ is defined in Eq. (38), $R_{\text {target, } j}$ is defined in Eq. (36), and $D_{\text {target, } k}$ is defined in Eq. (39). The adjustable parameter $b$ is used to dynamically balance the error between the reflectivity of the magnitude response and the dispersion response. The local Quasi-Newton method is employed to find the optimum of Eq. (40). The optimization results of the design of an FBG-based linear phase filter with a grating length of $31.1 \mathrm{~mm}$ are presented next. The solid curve of Fig. 14(a) shows the index modulation profile of an optimized FBG-based linear phase filter with a length of $31.1 \mathrm{~mm}$ using the hybrid Tabu algorithm; the corresponding group-delay response is shown as the solid curve in Fig. 14(b) and the corresponding reflection magnitude response is shown as the solid curve in Fig. 15. The index modulation profile (dotted curve of Fig. 14(a)), the group-delay response (dotted curve of Fig. 14(b)) and the reflection magnitude response (dotted curve of Fig. 15) of a 31.1$\mathrm{mm}$ long quadratic-sine apodized FBG-based linear phase filter obtained from the standard design method (without using optimization) (Kashyap, 1999) are also shown for comparison purpose. In Fig. 15, the almost-squared reflection magnitude response of the optimized FBG-based linear phase filter has a good sidelobe suppression level of $<-45 \mathrm{~dB}$ (which is acceptable for many practical applications), while the almost-squared reflection magnitude response of the unoptimized FBG-based linear phase filter has a very good sidelobe suppression level of $<-55 \mathrm{~dB}$. Fig. 14(b) shows that the in-band group delay ripple of the optimized linear phase filter is $<0.3$ ps (the solid curve), which is a lot flatter than that of the unoptimized linear phase filter (the dotted curve). It can thus be concluded that the

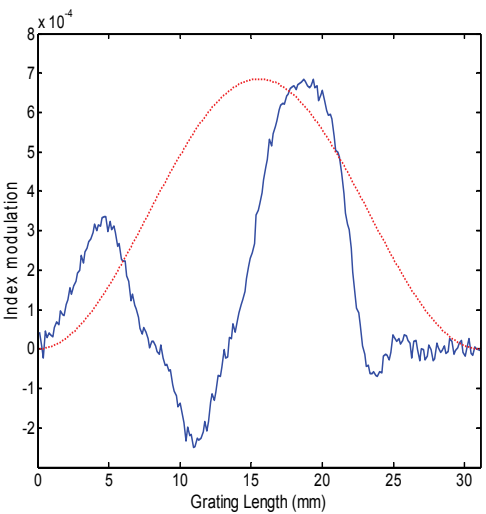

(a)

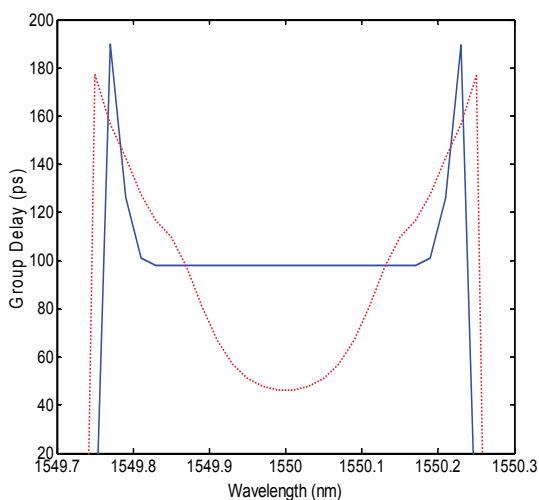

(b)

Fig. 14. Index modulation profile (solid curve) (a) and the corresponding group-delay response (solid curve) (b) of an optimized 31.1-mm long FBG-based linear phase filter using the hybrid Tabu algorithm. Index modulation profile (dotted curve) (a) and the corresponding group-delay response (dotted curve) (b) of a 31.1-mm long quadratic-sine apodized FBG using the standard design method (without using optimization). 
optimized FBG-based linear phase filter using the hybrid Tabu algorithm has linear phase characteristics and near-ideal squared reflection magnitude response which meet the design specifications. Table 4 also shows the performances of other optimized linear phase filters with shorter grating lengths of $17.1 \mathrm{~mm}$ and $25.8 \mathrm{~mm}$. The designed filter with the longest grating length of $31.1 \mathrm{~mm}$ has the smallest sidelobe level of $-45 \mathrm{~dB}$ while the designed filter with the shortest grating length of $17.1 \mathrm{~mm}$ has the largest sidelobe of $-35 \mathrm{~dB}$. However, the group delay ripple increases with the grating length of the designed filter. The designed filter with the longest grating length of $31.1 \mathrm{~mm}$ has the largest in-band group delay ripple of $0.3 \mathrm{ps}$ while the designed filter with the shortest grating length of $17.1 \mathrm{~mm}$ has the smallest group delay ripple of $0.1 \mathrm{ps}$. Thus a long grating length will yield a large sidelobe level but at the expense of a large group delay ripple. We can thus conclude that there is a trade-off between the maximum sidelobe value and the maximum in-band group delay ripple for a particular grating length.

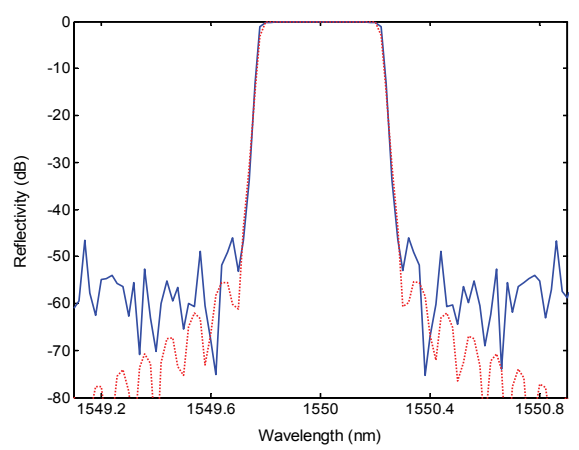

Fig. 15. The solid curve is the corresponding magnitude response of the index modulation profile (the solid curve of Fig. 14(a)) of a 31.1-mm long optimized linear phase filter obtained from the hybrid Tabu algorithm. The dotted curve is the corresponding magnitude response of the index modulation profile (the dotted curve of Fig. 14(a)) of a 31.1-mm long quadraticsine apodized FBG obtained from the standard design method (without using optimization).

\begin{tabular}{|c|c|c|}
\hline Grating length $(\mathrm{mm})$ & Maximum sidelobe value $(\mathrm{dB})$ & $\begin{array}{c}\text { Maximum in-band group } \\
\text { delay ripple (ps) }\end{array}$ \\
\hline 17.1 & -35 & 0.1 \\
\hline 25.8 & -37 & 0.2 \\
\hline 31.1 & -45 & 0.3 \\
\hline
\end{tabular}

Table 4. Performance comparison of three optimized FBG-based linear phase filters with different grating lengths using the hybrid Tabu algorithm.

Two main issues involved in the fabrication of the optimized index modulation (i.e., the "ac" index change) profile shown in the solid curve of Fig. 14(a) are described as follows. First, the fabrication process must be able to realize such a relatively complex index modulation profile along the grating length while maintaining an unperturbed " $\mathrm{dc}^{\prime}$ " index change along the grating length. The fabrication process required to realize this optimized index modulation profile is similar to that used in the fabrication of the optimized apodized index modulation profile shown in Fig. 11(a). This is because the fabrication of these two types of profiles is to produce some desired index modulation values corresponding to the grating positions while maintaining an unperturbed " $\mathrm{dc}$ " index change. To meet this first 
requirement, a well-developed technique of fiber dithering (which involves dithering of the fiber during the UV exposure) can be used (Asseh et al., 1997). In this method, the first step is to divide the whole grating into a number of sub-gratings. Each sub-grating inscribed by the fiber dithering will cause the index modulation depth to be averaged out. That is, if the offset of the fiber dithering is half of the grating period, the index modulation will be completely averaged out (i.e., no index modulation). Now if there is no offset of the fiber dithering from the phase mask, the index modulation will be maximum. Note that, to maintain an unperturbed ' $\mathrm{dc}$ ' index change along the grating length, all the sub-gratings must be exposed with the same total number of UV pulses (i.e., the exposure energy levels of all the sub-gratings are the same). Second, the fabrication process must be able to introduce some $\pi$ phase shifts (i.e., negative index modulation values) along the grating length (see solid curve of Fig. 14(a)). To meet this second requirement, the fiber should be moved relative to the phase mask (which is also a well-developed method), and this would enable the insertion of $\pi$ phase shifts into the gratings during the fabrication process (Asseh et al., 1997; Cole et al., 1995).

\section{Conclusion}

A hybrid Tabu algorithm has been presented for the synthesis and fabrication of FBG-based filters with specified frequency responses. The hybrid Tabu algorithm is a two-tier search that employs the global staged continuous Tabu search (SCTS) algorithm and a local optimization algorithm (i.e., the Quasi-Newton method). The first step of the hybrid Tabu algorithm (which employs the global SCTS algorithm) produces a "promising" grating structure which is then further optimized by the second stage of the hybrid Tabu algorithm (which employs the Quasi-Newton algorithm) to yield the final optimized solution or grating structure. In the hybrid Tabu algorithm, the synthesis problem of an FBG is formulated as an objective function which measures the error between the optimized reflection frequency response of an FBG based on the hybrid Tabu algorithm and the target frequency response, and the optimized solution or grating structure has the smallest error. The FBG-based filters are based on non-uniform FBGs with a wide variety of frequency responses. The transfer matrix method (TMM) has been employed to model a non-uniform FBG because it allows the magnitude and phase responses of a non-uniform FBG to be obtained with reasonably high accuracy. In TMM, a non-uniform FBG can be modeled using either the cascade of serially-connected uniform sub-gratings (this model is sufficient for obtaining the magnitude response when the phase response is not important) or the cascade of serially-connected apodized sub-gratings (this model must be used when both the magnitude and phase responses are important). The effectiveness of the hybrid Tabu algorithm has been demonstrated through the design and fabrication of 25-GHz-bandwidth FBG-based bandpass filters with near-squared bandpass responses and the design of 50GHz-bandwidth FBG-based linear phase filters with near-squared bandpass responses and flat group delay responses, which have potential application in optical communication systems operating in the $1550 \mathrm{~nm}$ wavelength window. For future work, the presented hybrid Tabu algorithm can be further developed into a powerful toolbox that can be efficiently and reliably used for the synthesis and fabrication of a wide variety of FBG-based filters with specified complex frequency responses to meet the increasing demand of sophisticated signal processing and filtering functions of the next generation of optical sensors and optical communication systems. Furthermore, the powerful design toolbox must be powerful enough for use in the diagnosis or characterization during and after fabrication of an FBG. 


\section{References}

Andre, J.; Siarry, P. \& Dognon, T. (2000). An improvement of the standard genetic algorithm fighting premature convergence in continuous optimization. Advances in Engineering Software, Vol. 32, No. 1, (December 2000), pp. 49-60. ISSN 0965-9978.

Asseh, A; Storoy, H; Sahlgren, B. E.; Sandgren, S. \& Stubbe R. (1997). A writing technique for long fiber Bragg gratings with complex reflectivity profiles. Journal of Lightwave Technology, Vol. 15, No. 8, (August 1997), pp. 1419-1424. ISSN 0733-8724.

Baskar, S.; Zheng, R. T.; Alphones, A.; Ngo, N. Q. \& Suganthan, P. N. (2005a). Particle swarm optimization for the design of low-dispersion fiber Bragg gratings. IEEE Photonics Technology Letters, Vol. 17, No. 3, (March 2005), pp. 615-617. ISSN 1041-1135.

Baskar, S.; Alphones, A.; Suganthan, P. N.; Ngo, N. Q. \& Zheng, R. T. (2005b). Design of optimal length low-dispersion FBG filter using covariance matrix adapted evolution. IEEE Photonics Technology Letters, Vol. 17, No. 10, (October 2005), pp. 2119-2121. ISSN 1041-1135.

Baskar, S.; Suganthan, P. N.; Ngo, N. Q.; Alphones, A. \& Zheng, R. T. (2006). Design of triangular FBG filter for sensor applications using covariance matrix adapted evolution algorithm. Optics Communications, Vol. 260, No. 2, (April 2006), pp. 716-722. ISSN 0030-4018.

Caucheteur, C.; Lhomme, F.; Chah, K.; Blondel, M. \& Megret, P. (2004). Fiber Bragg grating sensor demodulation technique by synthesis of grating parameters from its reflection spectrum. Optics Communications, Vol. 240, No. 4-6, (October, 2004), pp. 329-336. ISSN 0030-4018.

Chelouah, R. \& Siarry, P. (2000). Tabu search applied to global optimization. European Journal of Operational Research, Vol. 123, No. 2, (June 2000), pp. 256-270. ISSN 0377-2217

Cheng, H.-C. \& Lo, Y.-L. (2004). Arbitrary strain distribution measurement using a genetic algorithm approach for two fiber Bragg grating intensity spectra. Optics Communications, Vol. 239, No. 4-6, (September 2004), pp. 323-332. ISSN 0030-4018.

Cole, M. J.; Loh, W. H.; Laming, R. I.; Zervas, M. N. \& Barcelos, S. (1995). Moving fibre/phase mask-scanning beam technique for enhanced flexibility in producing fiber gratings with uniform phase mask. Electronics Letters, Vol. 31, No. 17, (August 1995), pp. 1488-1450. ISSN 0013-5194.

Dong, P.; Azana, J. \& Kirk, A. G. (2003). Synthesis of fiber Bragg grating parameters from reflectivity by means of a simulated annealing algorithm. Optics Communications, Vol. 228, No. 4-6, (December 2003), pp. 303-308. ISSN 0030-4018.

Erdogan, T. (1997). Fiber grating spectra. Journal of Lightwave Technology, Vol. 15, No. 8, (August 1997), pp. 1277-1294. ISSN 0733-8724.

Feced, R.; Zervas, M. N. \& Muriel, M. A. (1999). An efficient inverse scattering algorithm for the design of nonuniform fiber Bragg gratings. IEEE Journal in Quantum Electronics, Vol. 35, No. 8, (August 1999), pp. 1105-1115. ISSN 0018-9197.

Gill, A.; Peters, K. \& Studer, M. (2004). Genetic algorithm for the reconstruction of Bragg grating sensor strain profiles. Measurement Science and Technology, Vol. 15, No. 9, (September 2004), pp. 1877-1884. ISSN 0957-0233.

Glover, F. \& Laguna, M. (1998). Tabu search, Kluwer Academic Publishers, Boston. ISBN 0792381874. 
Hill, K. O. \& Meltz, G. (1997). Fiber Bragg grating technology: fundamentals and overview. Journal of Lightwave Technology, Vol. 15, No. 8, (August 1997), pp. 1263-1276. ISSN 0733-8724.

Ho, S. L.; Yang, S. Y.; Ni, G. Z. \& Wong, H. C. (2001). An improved tabu search for the global optimizations of electromagnetic devices. IEEE Transactions on Magnetics, Vol. 37, No. 5, (September 2001), pp. 3570-3574. ISSN 0018-9464.

Kashyap, R. (1999). Fiber Bragg gratings, Academic Press, ISBN 0-12-400560-8, San Diego.

Machado, J. M.; Yang, S.; Ho, S. L. \& Ni, P. (2001). A common tabu search algorithm for the global optimization of engineering problems. Computer Methods in Applied Mechanics and Engineering, Vol. 190, No. 26-27, (March 2001), pp. 3501-3510. ISSN 0045-7825.

Manos, S. \& Poladian, L. (2005). Multi-objective and constrained design of fibre Bragg gratings using evolutionary algorithms. Optics Express, Vol. 13, No. 19, (September 2005), pp. 7350-7364. ISSN \#8059.

Ngo, N. Q.; Zheng, R. T.; Tjin, S. C. \& Li, S. Y. (2004). Tabu search synthesis of cascaded fiber Bragg gratings for linear phase filters. Optics Communications, Vol. 241, No. 1-3, (November, 2004), pp. 79-85. ISSN 0030-4018.

Ngo, N. Q.; Zheng, R. T.; Ng, J. H.; Tjin, S. C. \& Binh, L. N. (2007). Optimization of fiber Bragg gratings using a hybrid optimization algorithm. Journal of Lightwave Technology, Vol. 25, No. 3, (March 2007), pp. 799-802. ISSN 0733-8724.

Othonos, A. \& Kalli, K. (1999). Fiber Bragg gratings: fundamentals and applications in telecommunications and sensing, Artech House, ISBN 0-89006-344-3, Boston.

Plougmann, N. \& Kristensen, M. (2004). Efficient iterative technique for designing Bragg gratings. Optics Letters, Vol. 29, No. 1, (January 2004), pp. 23-25. ISSN 0146-9592.

Poladian, L. (2000). Simple grating synthesis algorithm. Optics Letters, Vol. 25, No. 11, (June 2000), pp. 787-789. ISSN 0146-9592.

Rosenthal, A. \& Horowitz, M. (2003). Inverse scattering algorithm for reconstructing strongly reflecting fiber Bragg gratings. IEEE Journal in Quantum Electronics, Vol. 39, No. 8, (August 2003), pp. 1018-1026. ISSN 0018-9197.

Shanno, D. F. (1970). Conditioning of Quasi-Newton methods for function minimization. Mathematics of Computation, Vol. 24, no. 111, (July 1970), pp. 647-656. ISSN 00255718.

Siarry, P. \& Berthiau, G. (1997). Fitting of tabu search to optimize functions of continuous variables. International Journal for Numerical Methods in Engineering, Vol. 40, No. 13, (July 1997), pp. 2449-2457. ISSN 0029-5981.

Skaar, J. \& Risvik, K. M. (1998). A genetic algorithm for the inverse problem in synthesis of fiber gratings. Journal of Lightwave Technology, Vol. 16, No. 10, (October 1998), pp. 1928-1932. ISSN 0733-8724.

Skaar, J.; Wang, L. \& Erdogan, T. (2001). On the synthesis of fiber Bragg gratings by layer peeling. IEEE Journal in Quantum Electronics, Vol. 37, No. 2, (February 2001), pp. 165-173. ISSN 0018-9197.

Zheng, R. T.; Ngo, N. Q.; Binh, L. N. \& Tjin, S. C. (2004). Two-stage hybrid optimization of fiber Bragg gratings for design of linear phase filters. Journal of Optical Society of America A: Optics Image Science and Vision, Vol. 21, No. 12, (December 2004), pp. 2399-2405. ISSN 1084-7529. 
Zheng, R. T.; Ngo, N. Q.; Shum, P; Tjin, S. C. \& Binh, L. N. (2005). A staged continuous tabu search algorithm for the global optimization and its applications to the design of fiber Bragg gratings. Computational Optimization and Applications, Vol. 30, No. 3, (March 2005), pp. 319-335. ISSN 09266003.

\section{Appendix: List of test functions}

- $\quad F 1$ (1 variable): $f(x)=2(x-0.75)^{2}+\sin (5 \pi x-0.4 \pi)-0.125$, where $0 \leq x \leq 1$.

- $\quad F 3(1$ variable $): f(x)=-\sum_{i=1}^{5} i \sin [(i+1) x+i]$, where $-10 \leq x \leq 10$.

- Branin (2 variables): $f(x, y)=a\left(y-b x^{2}+c x-d\right)^{2}+h(1-g) \cos (x)+h$, where $a=1, b=\frac{5.1}{4 \pi^{2}}, c=\frac{5}{\pi}, d=6, h=10, g=\frac{1}{8 \pi}$.

- $\quad$ Goldprice (2 variables): $f(x, y)=\left[1+(x+y+1)^{2}\left(19-14 x+3 x^{2}-14 y+6 x y+3 y^{2}\right)\right]$

$$
\times\left[30+(2 x-3 y)^{2}\left(18-32 x+12 x^{2}+48 y-36 x y+27 y^{2}\right)\right],
$$

where $-2 \leq x \leq 2,-2 \leq y \leq 2$.

- $\quad$ Shubert1 and Shubert2 (2 variables):

$$
f(x, y)=\left\{\sum_{j=1}^{5} j \cdot \cos [(j+1) x+j]\right\} \times\left\{\sum_{j=1}^{5} j \cdot \cos [(j+1) y+j]\right\}+\beta\left[(x+1.42513)^{2}+(y+0.80032)^{2}\right]
$$

where $-10 \leq x, y \leq 10, \beta=0.5$ for Shuber1, and $\beta=1$ for Shuber2.

- Shubert (2 variables): $f\left(x_{1}, x_{2}\right)=\left\{\sum_{j=1}^{5} j \cdot \cos \left[(j+1) x_{1}+j\right]\right\} \times\left\{\sum_{j=1}^{5} j \cdot \cos \left[(j+1) x_{2}+j\right]\right\}$ where $-10 \leq x_{i} \leq 10, i=1,2$.

- Hartmann1 $\left(H_{3,4}\right)$ (3 variables):

$$
f(x)=-\sum_{i=1}^{4} c_{i} \exp \left[-\sum_{j=1}^{3} a_{i j}\left(x_{j}-p_{i j}\right)^{2}\right], \quad \text { where } 0<x_{j}<1 \text { for } j=1,2,3 \text {. }
$$

\begin{tabular}{|c|c|c|c|c|c|c|c|}
\hline \multirow{2}{*}{$i$} & \multicolumn{3}{|c|}{$a_{i j}$} & \multirow{2}{*}{$c_{i}$} & \multicolumn{3}{|c|}{$p_{i j}$} \\
\cline { 2 - 5 } \cline { 6 - 8 } & $j=1$ & $j=2$ & $j=3$ & & $j=1$ & $j=2$ & $j=3$ \\
\hline 1 & 3.0 & 10.0 & 30.0 & 1.0 & 0.3689 & 0.1170 & 0.2673 \\
\hline 2 & 0.1 & 10.0 & 35.0 & 1.2 & 0.4699 & 0.4387 & 0.7470 \\
\hline 3 & 3.0 & 10.0 & 30.0 & 3.0 & 0.1091 & 0.8732 & 0.5547 \\
\hline 4 & 0.1 & 10.0 & 35.0 & 3.2 & 0.0382 & 0.5743 & 0.8828 \\
\hline
\end{tabular}


- $\quad$ Brown1 (20 variables):

$$
f(x)=\left[\sum_{i \in J}\left(x_{i}-3\right)\right]^{2}+\sum_{i \in J}\left[10^{-3}\left(x_{i}-3\right)^{2}-\left(x_{i}-x_{i+1}\right)+\exp \left[20\left(x_{i}-x_{i+1}\right)\right]\right]
$$

where $J=\{1,3, \cdots, 19\},-1 \leq x_{i} \leq 4$ for $1 \leq i \leq 20$, and $x=\left[x_{1}, \cdots, x_{20}\right]^{T}$.

- Brown3 (20 variables): $f(x)=\sum_{i=1}^{19}\left[\left(x_{i}^{2}\right)^{\left(x_{i+1}^{2}+1\right)}+\left(x_{i+1}^{2}\right)^{\left(x_{i}^{2}+1\right)}\right]$

where $x=\left[x_{1}, \cdots, x_{20}\right]^{T}$ and $-1 \leq x_{i} \leq 4$ for $1 \leq i \leq 20$.

- $\quad$ F5n (20 variables):

$$
f(x)=\left(\frac{\pi}{20}\right) \times\left\{10 \sin ^{2}\left(\pi y_{1}\right)+\Sigma\left[\left(y_{i}-1\right)^{2} \times\left(1+10 \sin ^{2}\left(\pi y_{i+1}\right)\right)\right]+\left(y_{20}-1\right)^{2}\right\}
$$

where $y_{i}=1+0.25\left(x_{i}-1\right), x=\left[x_{1}, \cdots, x_{20}\right]^{T},-10 \leq x_{i} \leq 10$.

- $\quad F 10 n$ (20 variables):

$$
f(x)=\left(\frac{\pi}{20}\right) \times\left\{10 \sin ^{2}\left(\pi x_{1}\right)+\sum_{i=1}^{19}\left[\left(x_{i}-1\right)^{2} \times\left(1+10 \sin ^{2}\left(\pi x_{i+1}\right)\right)\right]+\left(x_{20}-1\right)^{2}\right\}
$$

where $x=\left[x_{1}, x_{2}, \cdots, x_{20}\right]^{T}$ and $-10 \leq x_{i} \leq 10$.

- $\quad F 15 n$ (20 variables):

$f(x)=\left(\frac{1}{10}\right)\left\{\sin ^{2}\left(3 \pi x_{1}\right)+\sum_{i=1}^{19}\left[\left(x_{i}-1\right)^{2}\left(1+\sin ^{2}\left(3 \pi x_{i+1}\right)\right)\right]+\left(\frac{1}{10}\right)\left(x_{20}-1\right)^{2}\left[1+\sin ^{2}\left(2 \pi x_{20}\right)\right]\right\}$ where $x=\left[x_{1}, x_{2}, \cdots, x_{20}\right]^{T}$ and $-10 \leq x_{i} \leq 10$. 


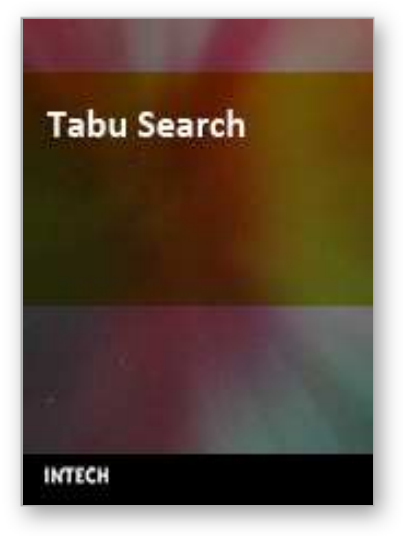

\author{
Tabu Search \\ Edited by Wassim Jaziri
}

ISBN 978-3-902613-34-9

Hard cover, 278 pages

Publisher I-Tech Education and Publishing

Published online 01, September, 2008

Published in print edition September, 2008

The goal of this book is to report original researches on algorithms and applications of Tabu Search to realworld problems as well as recent improvements and extensions on its concepts and algorithms. The book' Chapters identify useful new implementations and ways to integrate and apply the principles of Tabu Search, to hybrid it with others optimization methods, to prove new theoretical results, and to describe the successful application of optimization methods to real world problems. Chapters were selected after a careful review process by reviewers, based on the originality, relevance and their contribution to local search techniques and more precisely to Tabu Search.

\title{
How to reference
}

In order to correctly reference this scholarly work, feel free to copy and paste the following:

Nam Quoc Ngo and Ruitao Zheng (2008). Hybrid Tabu Algorithm for the Synthesis and Fabrication of Fiber Bragg Gratings, Tabu Search, Wassim Jaziri (Ed.), ISBN: 978-3-902613-34-9, InTech, Available from: http://www.intechopen.com/books/tabu_search/hybrid_tabu_algorithm_for_the_synthesis_and_fabrication_of_f iber_bragg_gratings

\section{INTECH}

open science | open minds

\section{InTech Europe}

University Campus STeP Ri Slavka Krautzeka 83/A 51000 Rijeka, Croatia Phone: +385 (51) 770447 Fax: +385 (51) 686166 www.intechopen.com

\section{InTech China}

Unit 405, Office Block, Hotel Equatorial Shanghai No.65, Yan An Road (West), Shanghai, 200040, China 中国上海市延安西路65号上海国际贵都大饭店办公楼 405 单元 Phone: +86-21-62489820

Fax: +86-21-62489821 
(C) 2008 The Author(s). Licensee IntechOpen. This chapter is distributed under the terms of the Creative Commons Attribution-NonCommercialShareAlike-3.0 License, which permits use, distribution and reproduction for non-commercial purposes, provided the original is properly cited and derivative works building on this content are distributed under the same license. 